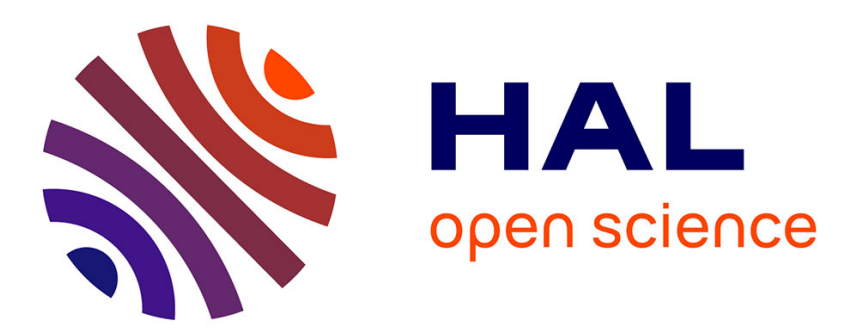

\title{
Uniformly accurate Particle-In-Cell method for the long time two-dimensional Vlasov-Poisson equation with strong magnetic field
}

\author{
Nicolas Crouseilles, Mohammed Lemou, Florian Méhats, Xiaofei Zhao
}

\section{- To cite this version:}

Nicolas Crouseilles, Mohammed Lemou, Florian Méhats, Xiaofei Zhao. Uniformly accurate ParticleIn-Cell method for the long time two-dimensional Vlasov-Poisson equation with strong magnetic field. Journal of Computational Physics, 2017, 346, pp.172-190. 10.1016/j.jcp.2017.06.011 . hal-01418976

\author{
HAL Id: hal-01418976 \\ https://hal.science/hal-01418976
}

Submitted on 18 Dec 2016

HAL is a multi-disciplinary open access archive for the deposit and dissemination of scientific research documents, whether they are published or not. The documents may come from teaching and research institutions in France or abroad, or from public or private research centers.
L'archive ouverte pluridisciplinaire HAL, est destinée au dépôt et à la diffusion de documents scientifiques de niveau recherche, publiés ou non, émanant des établissements d'enseignement et de recherche français ou étrangers, des laboratoires publics ou privés. 


\title{
Uniformly accurate Particle-In-Cell method for the long time two-dimensional Vlasov-Poisson equation with strong magnetic field
}

\author{
Nicolas Crouseilles ${ }^{\mathrm{b}, \mathrm{a}}$, Mohammed Lemou ${ }^{\mathrm{c}, \mathrm{a}, \mathrm{b}}$, Florian Méhats $^{\mathrm{a}, \mathrm{b}}$, Xiaofei Zhao $^{\mathrm{a}, *}$ \\ ${ }^{a}$ IRMAR, Université de Rennes I, France \\ ${ }^{b}$ INRIA-Rennes Bretagne Atlantique, IPSO Project, France \\ ${ }^{c}$ CNRS, Université de Rennes I, France
}

\begin{abstract}
In this work, we focus on the numerical resolution of the four dimensional phase space Vlasov-Poisson system subject to a strong external magnetic field. To do so, we consider a Particle-In-Cell based method, for which the characteristics are reformulated by means of the two-scale formalism, which is well-adapted to handle highly-oscillatory equations. Then, a numerical scheme is derived for the two-scale equations. The so-obtained scheme enjoys a uniform accuracy property, meaning that its accuracy does not depend on the small parameter. Several numerical results illustrate the capabilities of the method.
\end{abstract}

Keywords: Kinetic models, Vlasov-Poisson equation, Highly oscillatory, Four dimensional, Uniformly accurate, Particle-in-Cell, Two-scale formulation

\section{Introduction}

In plasma devices like tokamaks, when the charged particles are subject to a strong external magnetic field, a drift phenomenon will happen in the plane orthogonal to the magnetic field direction. As a model to describe this phenomenon, we consider in this paper the long time Vlasov-Poisson equation with a strong external magnetic field in the four dimensional phase space. Namely, we consider a distribution function $f^{\varepsilon}(t, \mathbf{x}, \mathbf{v})$ which depends on time $t \geq 0$, space $\mathbf{x}=\left(x_{1}, x_{2}\right)^{T} \in \mathbb{R}^{2}$ and velocity $\mathbf{v}=\left(v_{1}, v_{2}\right)^{T} \in \mathbb{R}^{2}$, which is solution to the following Vlasov-Poisson equation

$$
\begin{aligned}
& \partial_{t} f^{\varepsilon}(t, \mathbf{x}, \mathbf{v})+\frac{\mathbf{v}}{\varepsilon} \cdot \nabla_{\mathbf{x}} f^{\varepsilon}(t, \mathbf{x}, \mathbf{v})+\frac{1}{\varepsilon}\left(\mathbf{E}^{\varepsilon}(t, \mathbf{x})+\frac{1}{\varepsilon} \mathbf{v}^{\perp}\right) \cdot \nabla_{\mathbf{v}} f^{\varepsilon}(t, \mathbf{x}, \mathbf{v})=0 \\
& \nabla_{\mathbf{x}} \cdot \mathbf{E}^{\varepsilon}(t, \mathbf{x})=\rho^{\varepsilon}(t, \mathbf{x})-n_{i}, \quad \rho^{\varepsilon}(t, \mathbf{x}):=\int_{\mathbb{R}^{2}} f^{\varepsilon}(t, \mathbf{x}, \mathbf{v}) d \mathbf{v} \\
& f^{\varepsilon}(0, \mathbf{x}, \mathbf{v})=f_{0}(\mathbf{x}, \mathbf{v}), \quad \mathbf{x}, \mathbf{v} \in \mathbb{R}^{2}
\end{aligned}
$$

where $\mathbf{E}^{\varepsilon}$ is the electric field solution of the Poisson equation (1.1b), and where $f_{0}$ is a given initial data. We denote by $\mathbf{v}^{\perp}$ as

$$
\mathbf{v}^{\perp}=J \mathbf{v}=\left(v_{2},-v_{1}\right)^{T}, \quad \text { with } \quad J=\left(\begin{array}{cc}
0 & 1 \\
-1 & 0
\end{array}\right) .
$$

The real-valued scalar function $f^{\varepsilon}(t, \mathbf{x}, \mathbf{v})$ is the distribution function of electrons. The given parameters $\varepsilon>0$ is inversely proportional to the strength of the magnetic field and $n_{i}>0$ denotes the given ion density of the background which ensures global quasi-neutrality and hence the solvability of the Poisson equation (1.1b).

\footnotetext{
* Corresponding author.

Email addresses: nicolas.crouseilles@inria.fr (Nicolas Crouseilles), mohammed.lemou@univ-rennes1.fr (Mohammed Lemou), florian.mehats@univ-rennes1.fr (Florian Méhats), zhxfnus@gmail.com (Xiaofei Zhao) 
When the plasma is subject to a strong magnetic field, i.e. the parameter $\varepsilon$ becomes very small, the solution $f^{\varepsilon}(t, \mathbf{x}, \mathbf{v})$ of the Vlasov-Poisson equation (1.1) exhibits fast oscillations in time with wavelength $\mathcal{O}\left(\varepsilon^{2}\right)$. On the other side, there exists a slower dynamics of the so-called guiding-center, which is essentially given by the "E-cross- $B$ " drift velocity (see $[18,9]$ ). Thus, the system (1.1) is strongly multi-scale which implies strong restrictions on the numerical parameters when one wants to simulate the solution of (1.1).

When one is interested in the regime $\varepsilon \ll 1$, the asymptotic model is sufficient to describe the system in this case; and since it does not contain any stiff term, standard numerical methods can be employed to approximate it. The solution $f^{\varepsilon}$ of (1.1) has been proved to converge in a suitable sense towards $f$ when $\varepsilon$ goes to zero (see [12]) which solves

$$
\begin{aligned}
& \partial_{t} f(t, \mathbf{x}, \mathbf{v})+\mathbf{E}^{\perp}(t, \mathbf{x}) \cdot \nabla_{\mathbf{x}} f(t, \mathbf{x}, \mathbf{v})+\frac{1}{2} \nabla_{\mathbf{x}} \cdot \mathbf{E}(t, \mathbf{x}) \mathbf{v}^{\perp} \cdot \nabla_{\mathbf{v}} f(t, \mathbf{x}, \mathbf{v})=0 \\
& \nabla_{\mathbf{x}} \cdot \mathbf{E}(t, \mathbf{x})=\int_{\mathbb{R}^{2}} f(t, \mathbf{x}, \mathbf{v}) d \mathbf{v}-n_{i}
\end{aligned}
$$

As usual, the electric field $\mathbf{E}$ derives from a potential $\phi: \mathbf{E}=-\nabla_{\mathbf{x}} \phi$. By integrating (1.2a) with respect to $\mathbf{v} \in \mathbb{R}^{2}$, we further get the well-known guiding-center model

$$
\begin{aligned}
& \partial_{t} \rho(t, \mathbf{x})+\mathbf{E}^{\perp}(t, \mathbf{x}) \cdot \nabla_{\mathbf{x}} \rho(t, \mathbf{x})=0, \quad t>0, \mathbf{x} \in \mathbb{R}^{2} \\
& \nabla_{\mathbf{x}} \cdot \mathbf{E}(t, \mathbf{x})=\rho(t, \mathbf{x})-n_{i}, \\
& \rho(0, \mathbf{x})=\int_{\mathbb{R}^{2}} f_{0}(\mathbf{x}, \mathbf{v}) d \mathbf{v} .
\end{aligned}
$$

The unknown $\rho$ is the limit of the density $\rho^{\varepsilon}$ as $\varepsilon \rightarrow 0$ (see [20,2] for mathematical justification of this limit). As said before, these models are only valid in the asymptotic regime $\varepsilon \ll 1$.

From a numerical point of view, the passage from (1.1) to (1.3) has been explored by recent works, within the framework of Particle-In-Cell (PIC) method. The PIC framework (see [1, 15]) enables to focus on the construction of a numerical integrator in time of the characteristics equations of (1.1). In particular, in [12], an exponential time integrator has been proposed whereas in [10], the authors propose a high order IMEX type scheme. Both approaches are uniformly stable with respect to $\varepsilon$, and degenerate when $\varepsilon$ goes to zero to a consistent scheme with the asymptotic model (1.3). In particular, the numerical parameters can be chosen independently from $\varepsilon$. However, this so-called Asymptotic Preserving property (see [16]) does not guarantee the good behavior of the numerical scheme in the intermediate regime. The main goal of the present work is to construct a Uniformly Accurate (UA) numerical scheme with respect to $\varepsilon$. More precisely, in this work we will also use a Particle-In-Cell method, in which the characteristics equation will be reformulated using the two-scale formalism (as introduced in $[5,3]$ ). In addition to the uniform stability, this strategy enables to capture the highly oscillatory (in time) behavior of the solution, and to derive a uniformly accurate Particle-In-Cell method for $\varepsilon \in(0,1]$, which means that the accuracy of the scheme does not depend on $\varepsilon$.

The strategy we will use in this work relies on augmented characteristics equations, where the two time scales $t / \varepsilon^{2}$ and $t$ are separated. The solution of the original characteristics equation are recovered from the two-scale solution by evaluating it on the diagonal $\left(t, \tau=t / \varepsilon^{2}\right)$. Although the overall strategy follows the one introduced in $[5,3]$, several improvements have to be done to handle the problem (1.1). First, the model (1.1) under study involves two stiff terms: some transport terms (in $\mathbf{x}, \mathbf{v}$ ) of magnitude $1 / \varepsilon$ and a transport term in $\mathbf{v}$ of magnitude $1 / \varepsilon^{2}$. This contrasts with the previous study where only one stiffness of magnitude $1 / \varepsilon$ was involved, and naturally induces several difficulties. For instance, as we shall see, even after the filtering step, a nonlinear stiff term is still present in the reformulated equations. As this stiff term strongly depends on the self-consistent electric field, which is computed numerically, the numerical error is amplified by the stiffness factor $1 / \varepsilon$ and the uniform accuracy is difficult to obtain (contrary to what has been done in [17] where the analogous term was linear and then was considered implicit in time). To overcome this difficulty, a suitable change of unknown is performed, which enables to reformulate the two-scale model in 
order to fit with the theoretical framework of [3]. A final and important step is the suitable preparation of the initial data for the two-scale model. As proved in [3], a suitable choice of the initial condition (in the additional variable $\tau$ ) ensures that the time derivatives of the two-scale model are uniformly bounded with respect to $\varepsilon$. In the present work, a second order exponential integrator is used for the two-scale model, for which the local truncation error only depends on a second time derivative of the solution (whereas the second order scheme used in [3] induces the third time derivative). Then, a first order preparation of the initial data is sufficient to ensure its uniform boundedness and as a consequence, the uniform accuracy of the numerical scheme is obtained. Note that this first order correction should take into account the dependence in $\tau$ of the electric field and a tricky expansion with respect to $\varepsilon$ of the self-consistent electric field is then required.

It is worth mentionning that this UA PIC scheme is explicit in time and does not require any iterative algorithm. Moreover, as a byproduct of the uniform accuracy, the scheme has the good asymptotic behavior when $\varepsilon<<1$, since it is consistent with the asymptotic model (1.2). Several numerical tests are performed to validate and illustrate the capabilities of the UA PIC scheme in the 4 dimensional phase space setting. In particular, a few number of points in the additional $\tau$ direction are needed to produce accurate numerical results. Note that this $\tau$ direction is easily parallelized using MPI paradigm and the extra cost turns out to be negligible compared to the cost of a standard Particle-In-Cell method.

The rest of the paper is organized as follows. In the next Section, the UA PIC method is presented in details after a brief introduction of PIC methods. Then, some numerical results are given in Section 3 and conclusions are drawn in Section 4.

\section{Uniformly accurate particle-in-cell method}

In this section, we shall begin by briefly presenting the framework of the Particle-in-Cell (PIC) method where more details could be found in $[22,1]$, and then we shall introduce our uniformly accurate (UA) integrator towards the characteristics equation.

\subsection{Framework of PIC}

The starting point of the Particle-in-Cell method is to approximate the unknown distribution $f^{\varepsilon}(t, \mathbf{x}, \mathbf{v})$ of (1.1) by a sum of Dirac masses centred at $\left(\mathbf{x}_{k}(t), \mathbf{v}_{k}(t)\right)$ with weights $\omega_{k}>0$ for $k=1, \ldots, N_{p}$ and $N_{p} \in \mathbb{N}$ as

$$
f_{p}^{\varepsilon}(t, \mathbf{x}, \mathbf{v})=\sum_{k=1}^{N_{p}} \omega_{k} \delta\left(\mathbf{x}-\mathbf{x}_{k}(t)\right) \delta\left(\mathbf{v}-\mathbf{v}_{k}(t)\right), \quad t \geq 0, \mathbf{x}, \mathbf{v} \in \mathbb{R}^{2} .
$$

Inserting (2.1) into (1.1), in the sense of distribution, for $k=1, \ldots, N_{p}$, each particle obeys the characteristics equation

$$
\begin{aligned}
& \dot{\mathbf{x}}_{k}(t)=\frac{\mathbf{v}_{k}(t)}{\varepsilon}, \\
& \dot{\mathbf{v}}_{k}(t)=\frac{\mathbf{E}^{\varepsilon}\left(t, \mathbf{x}_{k}(t)\right)}{\varepsilon}+\frac{\mathbf{v}_{k}^{\perp}(t)}{\varepsilon^{2}}, \quad t>0, \\
& \mathbf{x}_{k}(0)=\mathbf{x}_{k, 0}, \quad \mathbf{v}_{k}(0)=\mathbf{v}_{k, 0} .
\end{aligned}
$$

The weight $\omega_{k}$ and initial values of the particles $\mathbf{x}_{k, 0}, \mathbf{v}_{k, 0}$ for $k=1, \ldots, N_{p}$ are prescribed according to the initial distribution $f_{0}(\mathbf{x}, \mathbf{v})$ given by $(1.1 \mathrm{c})$. To determine the weight, by integrating $(2.1)$ at $t=0$ in whole space we require

$$
\sum_{k=1}^{N_{p}} \omega_{k}=\int_{\mathbb{R}^{2} \times \mathbb{R}^{2}} f_{0}(\mathbf{x}, \mathbf{v}) d \mathbf{x} d \mathbf{v}
$$

Thus, a simple choice of uniform weight for all particles would be

$$
\omega_{k}=\frac{1}{N_{p}} \int_{\mathbb{R}^{2} \times \mathbb{R}^{2}} f_{0}(\mathbf{x}, \mathbf{v}) d \mathbf{x} d \mathbf{v}, \quad k=1, \ldots, N_{p} .
$$


The initialization of $\mathbf{x}_{k, 0}, \mathbf{v}_{k, 0}$ for $k=1, \ldots, N_{p}$ is a rather well-established and classical sampling issue. It could be done by the Monte Carlo type rejection sampling method as a general approach. The detailed process can be found in standard statistics textbooks. As a consequence, this approach introduces a noise of order $1 / \sqrt{N_{p}}($ see $[22])$. Note however that when $f_{0}$ is of variable separation form, i.e. $f_{0}(\mathbf{x}, \mathbf{v})=\chi_{1}(\mathbf{x}) \chi_{2}(\mathbf{v})$ where $\chi_{2}$ is a Gaussian, specific pseudo-random methods can be used. We omit the details here for brevity and refer the reader to $[1]$.

The characteristics equations for particles (2.2) are coupled to the Poisson equation (1.1b) through the electrical field $\mathbf{E}^{\varepsilon}$. Once the positions $\left\{\mathbf{x}_{k}(t)\right\}_{k=1, \ldots, N_{p}}$ of the particles are obtained at time $t>0$, one needs to evaluate the approximated density

$$
\rho_{p}^{\varepsilon}(t, \mathbf{x})=\sum_{k=1}^{N_{p}} \omega_{k} \delta\left(\mathbf{x}-\mathbf{x}_{k}(t)\right) \approx \rho^{\varepsilon}(t, \mathbf{x}) \quad \mathbf{x} \in \mathbb{R}^{2},
$$

then solve the Poisson equation

$$
\nabla_{\mathbf{x}} \cdot \mathbf{E}_{p}^{\varepsilon}(t, \mathbf{x})=\rho_{p}^{\varepsilon}(t, \mathbf{x})-n_{i}, \quad \mathbf{x} \in \mathbb{R}^{2},
$$

on a mesh grid of the spatial domain, with step size $\Delta x$. This provides an approximation $\mathbf{E}_{p}^{\varepsilon}(t, \mathbf{x}) \approx \mathbf{E}^{\varepsilon}(t, \mathbf{x})$. Finally, the values of $\mathbf{E}_{p}^{\varepsilon}\left(t, \mathbf{x}_{k}(t)\right)$ are interpolated at each particle position. In practice, the Dirac delta function $\delta(\mathbf{x})$ is replaced by smooth functions $S(\mathbf{x})$ (particle shape function). For example in 1D, it is approximated by the B-spline function $S^{m}(x)$ of order $m \in \mathbb{N}[22]$ :

$$
S^{0}(x):= \begin{cases}\frac{1}{\Delta x}, & |x| \leq \frac{\Delta x}{2}, \quad S^{m}(x):=\frac{1}{\Delta x} \int_{x-\frac{\Delta x}{2}}^{x+\frac{\Delta x}{2}} S^{m-1}(y) d y, \quad m \geq 1 . \\ 0, & \text { else, }\end{cases}
$$

The case in two dimensions is done by tensor product. The B-spline function $S^{m}$ is defined locally in space which is preferred from numerical point of view, but globally it is only a $C^{m-1}$ function for $m \geq 1$. A smooth but global basis has been considered in [15] to get high order accuracy. The classical PIC scheme is hence completed by a particle pusher for (2.2).

As is known, either the classical numerical methods or the asymptotic preserving methods would be problematic for solving oscillatory systems as (2.2) when $\varepsilon$ is in a certain regime, which in turns make the PIC scheme not efficient or less accurate for $\varepsilon \in(0,1]$. Thus, the main difficulty here is to solve the characteristic equation by some efficient integrator that works uniformly well for all $0<\varepsilon \leq 1$ and embed the integrator harmoniously into the PIC framework. We are going to apply the two-scale formulation approach developed in $[3,5]$ to derive a uniformly accurate PIC method.

\subsection{Two-scale formulation for characteristics}

This part is devoted to the derivation of the two-scale formulation of the highly-oscillatory ODE system (2.2). To do so, we follow two main steps: after a classical filtering step, the two-scale system can be obtained and then, a suitable change of unknown enables to recast the so-obtained two-scale system in a formulation like in [3]. Note that for simplicity of notation, in this subsection we shall omit the subscript $k$ for particles in (2.2).

First, we introduce a new variable $\mathbf{y}$ in order to filter out the main oscillation as

$$
\mathbf{y}(t)=\mathrm{e}^{-t J / \varepsilon^{2}} \mathbf{v}(t), \quad t \geq 0, \quad \text { with } \quad \mathrm{e}^{-t J}=\left(\begin{array}{cc}
\cos t & -\sin t \\
\sin t & \cos t
\end{array}\right)
$$

so that the characteristic equation (2.2) becomes

$$
\begin{aligned}
\dot{\mathbf{x}}(t) & =\frac{1}{\varepsilon} \mathrm{e}^{t J / \varepsilon^{2}} \mathbf{y}(t), \\
\dot{\mathbf{y}}(t) & =\frac{1}{\varepsilon} \mathrm{e}^{-t J / \varepsilon^{2}} \mathcal{E}\left(t, t / \varepsilon^{2}, \mathbf{x}(t)\right), \\
\mathbf{x}(0) & =\mathbf{x}_{0}, \quad \mathbf{y}(0)=\mathbf{v}_{0} .
\end{aligned}
$$


Then, we derive a suitable two-scale formulation by separating the fast scale $\tau=t / \varepsilon^{2}$ from the slow one $t$, as already done in $[5,3,6,17]$. We consider an augmented system of equations satisfied by the augmented solution $\left(X_{k}(t, \tau), Y_{k}(t, \tau)\right)\left(k=1, \ldots, N_{p}\right)$ which is periodic with respect to $\tau$ (of period $2 \pi$ ), and which coincides with the original solution $\left(\mathbf{x}_{k}(t), \mathbf{y}_{k}(t)\right)\left(k=1, \ldots, N_{p}\right)$ on the diagonal $\tau=t / \varepsilon^{2}$

$$
\mathbf{x}_{k}(t)=X_{k}\left(t, \frac{t}{\varepsilon^{2}}\right), \quad \mathbf{y}_{k}(t)=Y_{k}\left(t, \frac{t}{\varepsilon^{2}}\right), \quad t \geq 0, \quad k=1, \ldots, N_{p} .
$$

The two-scale equation satisfed by $\left(X_{k}(t, \tau), Y_{k}(t, \tau)\right)$ reads (removing the subscript $k$ )

$$
\begin{aligned}
\partial_{t} X(t, \tau)+\frac{1}{\varepsilon^{2}} \partial_{\tau} X(t, \tau) & =\frac{1}{\varepsilon} \mathrm{e}^{\tau J} Y(t, \tau), \\
\partial_{t} Y(t, \tau)+\frac{1}{\varepsilon^{2}} \partial_{\tau} Y(t, \tau) & =\frac{1}{\varepsilon} \mathrm{e}^{-\tau J} \mathcal{E}(t, \tau, X(t, \tau)),
\end{aligned}
$$

where the electric field is given by

$$
\nabla_{\mathbf{x}} \cdot \mathcal{E}(t, \tau, \mathbf{x}) \approx \sum_{\ell=1}^{N_{p}} \omega_{\ell} \delta\left(\mathbf{x}-X_{\ell}(t, \tau)\right)-n_{i}
$$

The augmented equation (2.7) are to be supplemented with the following initial data

$$
X(0,0)=\mathbf{x}(0), \quad Y(0,0)=\mathbf{y}(0) .
$$

Note that we can recover the original solution of (2.2) as

$$
\mathbf{x}(t)=X\left(t, \frac{t}{\varepsilon^{2}}\right), \quad \mathbf{v}(t)=\mathrm{e}^{t J / \varepsilon^{2}} Y\left(t, \frac{t}{\varepsilon^{2}}\right), \quad t \geq 0 .
$$

Solving (2.7) requires to solve numerically the augmented Poisson equation (2.8). Then, at each time, an approximation error of $\mathcal{E}(t, \tau, \mathbf{x})$ will be introduced in (2.7). This error (which depends on the number of particles $N_{p}$ and the mesh size $\Delta x$ in $\mathbf{x}$-space) is amplified by the factor $1 / \varepsilon$ on the right hand side of (2.7b), due to the diffusion scaling. As a matter of fact, the extra error apart from the time discretization will make the scheme non-uniformly accurate for $\varepsilon \in(0,1]$. Note that this lack of accuracy does not occur when $\mathcal{E}$ is given or explicitely depends on the solution. This later case corresponds to [17].

To overcome this essential difficulty, we seek for a suitable reformulation of (2.7). Let us introduce two new unknown functions $U_{ \pm}(t, \tau)$ :

$$
U_{+}(t, \tau):=X(t, \tau)+\varepsilon J \mathrm{e}^{\tau J} Y(t, \tau), \quad U_{-}(t, \tau):=-\varepsilon J Y(t, \tau), \quad t \geq 0, \tau \in[0,2 \pi] .
$$

The unknown $(X(t, \tau), Y(t, \tau))$ is obtained by the inverse change of variables

$$
X(t, \tau)=\left(U_{+}(t, \tau)+\mathrm{e}^{\tau J} U_{-}(t, \tau)\right), \quad Y(t, \tau)=\frac{1}{\varepsilon} J U_{-}(t, \tau) .
$$

Combining (2.9) with (2.7) leads to

$$
\partial_{t} U_{ \pm}+\frac{1}{\varepsilon^{2}} \partial_{\tau} U_{ \pm}=\mathcal{F}_{ \pm}\left(t, \tau, U_{+}, U_{-}\right)
$$

with

$$
\mathcal{F}_{+}\left(t, \tau, U_{+}, U_{-}\right):=J \mathcal{E}\left(t, \tau, U_{+}+\mathrm{e}^{\tau J} U_{-}\right), \mathcal{F}_{-}\left(t, \tau, U_{+}, U_{-}\right):=-J \mathrm{e}^{-\tau J} \mathcal{E}\left(t, \tau, U_{+}+\mathrm{e}^{\tau J} U_{-}\right),
$$

with the initial condition

$$
U_{+}(0,0)=\mathbf{x}_{0}+\varepsilon J \mathbf{v}_{0}=: \mathbf{u}_{+}^{0}, \quad U_{-}(0,0)=-\varepsilon J \mathbf{v}_{0}=: \mathbf{u}_{-}^{0},
$$


$\mathbf{x}_{0}, \mathbf{v}_{0}$ being the initial condition of the original problem $(2.2)$. Note that $\mathcal{E}$ is computed from $\left(U_{ \pm}\right)_{\ell}, \ell=$ $1, \ldots, N_{p}$ by the Poisson equation

$$
\nabla_{\mathbf{x}} \cdot \mathcal{E}(t, \tau, \mathbf{x})=\sum_{\ell=1}^{N_{p}} \omega_{\ell} \delta\left(\mathbf{x}-\left(U_{+}+\mathrm{e}^{\tau J} U_{-}\right)_{\ell}\right) .
$$

Again, from the solution of (2.11), the solution of (2.2) is recovered via the following relations

$$
\mathbf{x}(t)=U_{+}\left(t, t / \varepsilon^{2}\right)+\mathrm{e}^{t J / \varepsilon^{2}} U_{-}\left(t, t / \varepsilon^{2}\right), \quad \mathbf{v}(t)=\frac{1}{\varepsilon} \mathrm{e}^{t J / \varepsilon^{2}} J U_{-}\left(t, t / \varepsilon^{2}\right) .
$$

Comparing to (2.7), now (2.11) has bounded coefficients in front of the nonlinearity and it enters in the framework studied [3]. In [3], it is proven that, the initial condition $U_{ \pm}(t=0, \tau)$ has to be chosen under a suitable expansion to ensure that the time derivatives of $U_{ \pm}$are uniformly bounded with respect to $\varepsilon$. This is of importance when one applies a numerical discretization to (2.11) since a uniform accuracy property can then proved for the numerical scheme (see $[3,17,6,5,4])$. The construction of the suitable initial data $U_{ \pm}(t=0, \tau)$ is based on a Chapman-Enskog type expansion and is detailed in the next subsection.

\subsection{Derivation of the initial condition}

Following the strategy proposed in $[3,17,6,5,4]$, we will derive a suitable initial condition for $(2.11)$. To do so, we will perform a Chapman-Enskog expansion of $U_{ \pm}(t, \tau)$ so that $U_{ \pm}(0,0)=\mathbf{u}_{ \pm}^{0}$.

Chapman-Enskog expansion of $U_{ \pm}$. We denote $L u(\tau)=\partial_{\tau} u(\tau)$ for some periodic function $u(\tau)$ on $[0,2 \pi]$ and introduce the average operator $\Pi$ as

$$
\Pi u=\frac{1}{2 \pi} \int_{0}^{2 \pi} u(s) d s
$$

Then $L$ is invertible on the set of periodic functions with zero average, i.e. for $u(\tau)$ with $\Pi u=0$,

$$
L^{-1} u(\tau)=(I-\Pi) \int_{0}^{\tau} u(s) d s .
$$

We introduce the decomposition

$$
U_{ \pm}(t, \tau)=\underline{U}_{ \pm}(t)+\mathbf{h}_{ \pm}(t, \tau), \quad \text { with } \quad \underline{U}_{ \pm}(t)=\Pi U_{ \pm}(t, \tau),
$$

then we have the equation for the macro part of the solution

$$
\partial_{t} \underline{U}_{ \pm}(t)=\Pi \mathcal{F}_{ \pm}\left(t, \tau, \underline{U}_{+}(t)+\mathbf{h}_{+}(t, \tau), \underline{U}_{-}(t)+\mathbf{h}_{-}(t, \tau)\right),
$$

and the equation for the micro part

$$
\partial_{t} \mathbf{h}_{ \pm}(t, \tau)+\frac{1}{\varepsilon^{2}} L \mathbf{h}_{ \pm}(t, \tau)=(I-\Pi) \mathcal{F}_{ \pm}\left(t, \tau, \underline{U}_{+}(t)+\mathbf{h}_{+}(t, \tau), \underline{U}_{-}(t)+\mathbf{h}_{-}(t, \tau)\right)
$$

By inverting $L$ in the micro part equation (2.16), we get

$$
\mathbf{h}_{ \pm}(t, \tau)=\varepsilon^{2} A \mathcal{F}_{ \pm}\left(t, \tau, \underline{U}_{+}(t)+\mathbf{h}_{+}(t, \tau), \underline{U}_{-}(t)+\mathbf{h}_{-}(t, \tau)\right)-\varepsilon^{2} L^{-1} \partial_{t} \mathbf{h}_{ \pm}(t, \tau),
$$

where $A:=L^{-1}(I-\Pi)$. By assuming $\partial_{t} \mathbf{h}_{ \pm}=O(1)$, we get $\mathbf{h}_{ \pm}=O\left(\varepsilon^{2}\right)$. By further assuming $\partial_{t}^{2} \mathbf{h}_{ \pm}=O(1)$, we get a fourth order expansion of $U_{ \pm}$as

$$
U_{ \pm}(t, \tau)=\underline{U}_{ \pm}(t)+\varepsilon^{2} A \mathcal{F}_{ \pm}\left(t, \tau, \underline{U}_{+}(t), \underline{U}_{-}(t)\right)+O\left(\varepsilon^{4}\right),
$$


where we recall that $\mathcal{F}_{ \pm}\left(t, \tau, \underline{U}_{+}(t), \underline{U}_{-}(t)\right)$ are given by

$$
\begin{gathered}
\mathcal{F}_{+}\left(t, \tau, \underline{U}_{+}(t), \underline{U}_{-}(t)\right)=J \mathcal{E}\left(t, \tau, \underline{U}_{+}+\mathrm{e}^{\tau J} \underline{U}_{+}\right), \\
\mathcal{F}_{-}\left(t, \tau, \underline{U}_{+}(t), \underline{U}_{-}(t)\right)=-J \mathrm{e}^{-\tau J} \mathcal{E}\left(t, \tau, \underline{U}_{+}+\mathrm{e}^{\tau J} \underline{U}_{+}\right) .
\end{gathered}
$$

One has to point out that the electric field $\mathcal{E}$ is computed through the Poisson equation (2.14), and then evaluated at $\left(\underline{U}_{+}+\mathrm{e}^{\tau J} \underline{U}_{+}\right)$. To get an expansion of $U_{ \pm}$as a function of $\underline{U}_{ \pm}$, one then needs to also expand the electric field $\mathcal{E}$ solution of (2.14). To do so, we approximate $U_{ \pm}$in the right hand side of (2.14) by $\underline{U}_{ \pm}$. We will see that this approximation provides a second order approximation of $\mathcal{E}$, as required from the expansion (2.17).

Denoting by $X^{1 s t}$ the following quantity

$$
X^{1 s t}(t, \tau):=\underline{U}_{+}(t)+e^{\tau J} \underline{U}_{-}(t),
$$

one has $X=X^{1 s t}+\mathcal{O}\left(\varepsilon^{2}\right)$. Indeed, on the one side, a Chapman-Enskog expansion of $X$ solution of (2.7) reads

$$
X(t, \tau)=\underline{X}(t)+\varepsilon L^{-1} \mathrm{e}^{\tau J} Y(t, \tau)+\mathcal{O}\left(\varepsilon^{2}\right)=\underline{X}(t)-\varepsilon J \mathrm{e}^{\tau J} \underline{Y}(t)+\mathcal{O}\left(\varepsilon^{2}\right) .
$$

On the other side, one can express $X^{1 s t}$ defined in (2.20) in terms of $\underline{X}$ and $\underline{Y}$ using (2.9)

$$
X^{1 s t}(t, \tau)=\underline{X}(t)+\varepsilon J \Pi\left(\mathrm{e}^{\tau J} Y(t, \tau)\right)-\varepsilon e^{\tau J} J \underline{Y}(t)=\underline{X}(t)-\varepsilon J e^{\tau J} \underline{Y}(t)+\mathcal{O}\left(\varepsilon^{2}\right) .
$$

We deduce from the two last equation that $X=X^{1 s t}+\mathcal{O}\left(\varepsilon^{2}\right)$.

Then, using the Poisson equation (2.8), one can compute $\mathcal{E}^{1 s t}$

$$
\nabla_{\mathbf{x}} \cdot \mathcal{E}^{1 s t}(t, \tau, \mathbf{x}) \approx \sum_{k=1}^{N_{p}} \omega_{k} \delta\left(\mathbf{x}-X_{k}^{1 s t}(t, \tau)\right)-n_{i},
$$

and we deduce $\mathcal{E}(t, \tau, \mathbf{x})=\mathcal{E}^{1 s t}(t, \tau, \mathbf{x})+\mathcal{O}\left(\varepsilon^{2}\right)$. Finally $\mathcal{E}^{1 s t}$ enables to get $\mathcal{F}_{ \pm}^{1 s t}$ as in (2.18)-(2.19):

$$
\begin{gathered}
\mathcal{F}_{+}^{1 s t}\left(t, \tau, \underline{U}_{+}(t), \underline{U}_{-}(t)\right)=J \mathcal{E}^{1 s t}\left(t, \tau, \underline{U}_{+}(t)+e^{\tau J} \underline{U}_{-}(t)\right), \\
\mathcal{F}_{-}^{1 s t}\left(t, \tau, \underline{U}_{+}(t), \underline{U}_{-}(t)\right)=-J \mathrm{e}^{-\tau J} \mathcal{E}^{1 s t}\left(t, \tau, \underline{U}_{+}(t)+e^{\tau J} \underline{U}_{-}(t)\right) .
\end{gathered}
$$

We end up by defining $U^{2 n d}$ as

$$
U_{ \pm}^{2 n d}(t, \tau)=\underline{U}_{ \pm}(t)+\varepsilon^{2} A \mathcal{F}_{ \pm}^{1 s t}\left(t, \tau, \underline{U}_{+}(t), \underline{U}_{-}(t)\right) .
$$

Link with the original initial condition $\mathbf{u}_{ \pm}^{0}$. Now, we need to compute $\underline{U}_{ \pm}$so that $U_{ \pm}^{2 n d}(0,0)=\mathbf{u}_{ \pm}^{0}$ where $U^{2 n d}$ is defined by (2.24). Note that $\mathbf{u}_{ \pm}^{0}$ is defined by (2.13) as a function of $\left(\mathbf{x}_{0}, \mathbf{v}_{0}\right)$ which is the initial data of the original problem. Evaluating (2.24) at $t=\tau=0$ and using $U_{ \pm}^{2 n d}(0,0)=\mathbf{u}_{ \pm}^{0}$, one has directly $\mathbf{u}_{ \pm}^{0}-\underline{U}_{ \pm}(t=0)=\mathcal{O}\left(\varepsilon^{2}\right)$. Then, we can rewrite $(2.24)$ at $t=0$ as

$$
U_{ \pm}^{2 n d}(0, \tau)=\underline{U}_{ \pm}(0)+h_{ \pm}^{1 s t}(\tau)+\mathcal{O}\left(\varepsilon^{4}\right), \text { with } h_{ \pm}^{1 s t}(\tau)=\varepsilon^{2} A \mathcal{F}_{ \pm}^{1 s t}\left(0, \tau, \mathbf{u}_{+}^{0}, \mathbf{u}_{-}^{0}\right)
$$

and $\mathcal{F}_{ \pm}^{1 s t}$ defined by (2.22)-(2.23). Using again the condition $U_{ \pm}^{2 n d}(0,0)=\mathbf{u}_{ \pm}^{0}$, we get from $(2.25)$ at $\tau=0$ : $\underline{U}_{ \pm}(0)=\mathbf{u}_{ \pm}^{0}-h_{ \pm}^{1 s t}(0)$. Replacing $\underline{U}_{ \pm}(0)$ by this latter expression in (2.25) leads to

$$
U_{ \pm}^{2 n d}(0, \tau)=\mathbf{u}_{ \pm}^{0}-h_{ \pm}^{1 s t}(0)+h_{ \pm}^{1 s t}(\tau), \text { with } h_{ \pm}^{1 s t}(\tau):=\varepsilon^{2} A \mathcal{F}_{ \pm}^{1 s t}\left(0, \tau, \mathbf{u}_{+}^{0}, \mathbf{u}_{-}^{0}\right),
$$

with

$$
\begin{gathered}
\mathcal{F}_{+}^{1 s t}\left(0, \tau, \mathbf{u}_{+}^{0}, \mathbf{u}_{-}^{0}\right)=J \mathcal{E}^{1 s t}\left(0, \tau, \mathbf{u}_{+}^{0}+e^{\tau J} \mathbf{u}_{-}^{0}\right) \\
\mathcal{F}_{-}^{1 s t}\left(0, \tau, \mathbf{u}_{+}^{0}, \mathbf{u}_{-}^{0}\right)=-J e^{-\tau J} \mathcal{E}^{1 s t}\left(0, \tau, \mathbf{u}_{+}^{0}+e^{\tau J} \mathbf{u}_{-}^{0}\right),
\end{gathered}
$$


where $\mathcal{E}^{1 s t}$ is computed with the following Poisson equation

$$
\nabla_{\mathbf{x}} \cdot \mathcal{E}^{1 s t}(0, \tau, \mathbf{x})=\sum_{k=1}^{N_{p}} \omega_{k} \delta\left(\mathbf{x}-\left(\left(\mathbf{u}_{+}^{0}\right)_{k}+e^{\tau J}\left(\mathbf{u}_{-}^{0}\right)_{k}\right)\right)-n_{i},
$$

and where $\mathbf{u}_{+}^{0}=\mathbf{x}_{0}+\varepsilon J \mathbf{v}_{0}, \mathbf{u}_{-}^{0}=-\varepsilon J \mathbf{v}_{0}$.

We conclude this part with the following proposition which ensures that the solution of the two-scale equations (2.11) with the suitable initial condition (2.26) is smooth with respect to $\varepsilon$.

Proposition 2.1. Let us consider the following problem satisfied by $U_{ \pm}(t, \tau), t \in[0, T], T>0, \tau \in[0,2 \pi]$

$$
\partial_{t} U_{ \pm}+\frac{1}{\varepsilon^{2}} \partial_{\tau} U_{ \pm}=\mathcal{F}_{ \pm}\left(t, \tau, U_{+}, U_{-}\right),
$$

with the initial condition defined by (2.26) and the above notations. Then, there exists a constant $C>0$ independent of $\varepsilon$ such that,

$$
\left\|\partial_{t}^{k} U_{ \pm}\right\|_{L_{t, \tau}^{\infty}} \leq C, \quad k=0,1,2 .
$$

The proof will not be done here but can be obtained in a analogous way as in [3].

\subsection{An exponential integrator}

Now we present a numerical scheme for the discretization of the characteristics (2.11). We shall integrate the two-scale equation (2.11) by an exponential integrator.

Let $\Delta t>0$ be the time step and denote $t_{n}=n \Delta t$ for $n \geq 0$. Discretizating the $\tau$-direction as $\tau_{j}=$ $j \Delta \tau, j=0,1, \ldots, N_{\tau}$, with $\Delta \tau=2 \pi / N_{\tau}$ and $N_{\tau}$ some positive even integer, and applying the Fourier transform to $(2.11)$ on $[0,2 \pi]$, we get for $\ell=-N_{\tau} / 2, \ldots, N_{\tau} / 2-1$,

$$
\frac{d}{d t} \widehat{\left(U_{ \pm}\right)_{\ell}}(t)+\frac{i \ell}{\varepsilon^{2}} \widehat{\left(U_{ \pm}\right)_{\ell}}(t)=\widehat{\left(\mathcal{F}_{ \pm}\right)_{\ell}}(t), \quad t>0,
$$

where

$$
U_{ \pm}(t, \tau)=\sum_{\ell=-N_{\tau} / 2}^{N_{\tau} / 2-1} \widehat{\left(U_{ \pm}\right)_{\ell}}(t) \mathrm{e}^{i \ell \tau}, \quad \mathcal{F}_{ \pm}\left(t, \tau, U_{+}, U_{-}\right)=\sum_{\ell=-N_{\tau} / 2}^{N_{\tau} / 2-1} \widehat{\left(\mathcal{F}_{ \pm}\right)}(t) \mathrm{e}^{i \ell \tau}
$$

Integrating (2.28) from $t_{n}$ to $t_{n+1}(n \geq 0)$, we get

$$
\widehat{\left(U_{ \pm}\right)_{\ell}}\left(t_{n+1}\right)=\mathrm{e}^{-\frac{i \ell \Delta t}{\varepsilon^{2}}} \widehat{\left(U_{ \pm}\right)_{\ell}}\left(t_{n}\right)+\int_{0}^{\Delta t} \mathrm{e}^{-\frac{i \ell}{\varepsilon^{2}}(\Delta t-s)} \widehat{\left(\mathcal{F}_{ \pm}\right)_{\ell}}\left(t_{n}+s\right) d s .
$$

We approximate the above integral by the following quadrature for $n \geq 1$,

$$
\begin{aligned}
\widehat{\left(U_{ \pm}\right)_{\ell}}\left(t_{n+1}\right) & \approx \mathrm{e}^{-\frac{i \ell \Delta t}{\varepsilon^{2}}} \widehat{\left(U_{ \pm}\right)_{\ell}}\left(t_{n}\right)+\int_{0}^{\Delta t} \mathrm{e}^{-\frac{i \ell}{\varepsilon^{2}}(\Delta t-s)}\left(\widehat{\left(\mathcal{F}_{ \pm}\right)_{\ell}}\left(t_{n}\right)+s \frac{d}{d t} \widehat{\left(\mathcal{F}_{ \pm}\right)_{\ell}}\left(t_{n}\right)\right) d s \\
& \approx \mathrm{e}^{-\frac{i \ell \Delta t}{\varepsilon^{2}}} \widehat{\left(U_{ \pm}\right)_{\ell}}\left(t_{n}\right)+p_{\ell} \widehat{\left(\mathcal{F}_{ \pm}\right)_{\ell}}\left(t_{n}\right)+q_{\ell} \frac{1}{\Delta t}\left(\widehat{\left(\mathcal{F}_{ \pm}\right)_{\ell}}\left(t_{n}\right)-\widehat{\left(\mathcal{F}_{ \pm}\right)_{\ell}}\left(t_{n-1}\right)\right)
\end{aligned}
$$

where

$$
\begin{gathered}
p_{\ell}:=\int_{0}^{\Delta t} \mathrm{e}^{-\frac{i \ell}{\varepsilon^{2}}(\Delta t-s)} d s= \begin{cases}\frac{i \varepsilon^{2}}{\ell}\left(\mathrm{e}^{-\frac{i \ell \Delta t}{\varepsilon^{2}}}-1\right), & \ell \neq 0, \\
\Delta t, & \ell=0,\end{cases} \\
q_{\ell}:=\int_{0}^{\Delta t} \mathrm{e}^{-\frac{i \ell}{\varepsilon^{2}}(\Delta t-s)} s d s= \begin{cases}\frac{\varepsilon^{2}}{\ell^{2}}\left(\varepsilon^{2}-\varepsilon^{2} \mathrm{e}^{-\frac{i \ell \Delta t}{\varepsilon^{2}}}-i \ell \Delta t\right), & \ell \neq 0, \\
\frac{\Delta t^{2}}{2}, & \ell=0 .\end{cases}
\end{gathered}
$$


For the first iteration, the starting value $\widehat{\left(U_{ \pm}\right)_{\ell}}\left(t_{1}\right)$ (with $\left.t_{1}=\Delta t\right)$ is computed using a prediction-correction scheme. The first order prediction writes

$$
\left.{\widehat{\left(U_{ \pm}\right)_{\ell}}}_{\ell}^{*}\left(t_{1}\right):=\mathrm{e}^{-\frac{i \ell \Delta t}{\varepsilon^{2}}} \widehat{\left(U_{ \pm}\right)_{\ell}}(0)+p_{\ell} \widehat{(\mathcal{F}}_{ \pm}\right)_{\ell}(0)
$$

which gives a prediction of the nonlinearity at $t_{1}=\Delta t$ as

$$
\mathcal{F}_{ \pm}\left(t_{1}, \tau, U_{+}^{*}\left(t_{1}, \tau\right), U_{-}^{*}\left(t_{1}, \tau\right)\right)=\sum_{\ell=-N_{\tau} / 2}^{N_{\tau} / 2-1}{\widehat{\left(\mathcal{F}_{ \pm}\right)}}_{\ell}^{*}\left(t_{1}\right) \mathrm{e}^{i \ell \tau}
$$

Then, we do a correction step

$$
{\widehat{\left(U_{ \pm}\right)_{\ell}}}_{\ell}\left(t_{1}\right) \approx \mathrm{e}^{-\frac{i \ell \Delta t}{\varepsilon^{2}}} \widehat{\left(U_{ \pm}\right)_{\ell}}(0)+p_{\ell}{\widehat{\left(\mathcal{F}_{ \pm}\right)_{\ell}}}_{(0)}+q_{\ell} \frac{1}{\Delta t}\left({\widehat{\left(\mathcal{F}_{ \pm}\right)_{\ell}}}^{*}\left(t_{1}\right)-{\widehat{\left(\mathcal{F}_{ \pm}\right)}}_{\ell}(0)\right)
$$

At each time level $t_{n}(n \geq 0)$, when the approximated $U_{ \pm}\left(t_{n}, \tau\right)$ is obtained as above, we get

$$
X\left(t_{n}, \tau\right)=U_{+}\left(t_{n}, \tau\right)+\mathrm{e}^{\tau J} U_{-}\left(t_{n}, \tau\right), \quad t \geq 0, \tau \in[0,2 \pi] .
$$

and we compute

$$
\mathcal{E}\left(t_{n}, \tau, \mathbf{x}\right)=-\nabla_{\mathbf{x}} \Phi^{\varepsilon}\left(t_{n}, \tau, \mathbf{x}\right), \quad-\Delta \Phi^{\varepsilon}\left(t_{n}, \tau, \mathbf{x}\right) \approx \sum_{k=1}^{N_{p}} \omega_{k} \delta\left(\mathbf{x}-X_{k}\left(t_{n}, \tau\right)\right)-n_{i}
$$

then the evaluation of nonlinearity $\mathcal{F}_{ \pm}$follows from $(2.12)$

$$
\begin{aligned}
& \mathcal{F}_{+}\left(t_{n}, \tau, U_{+}\left(t_{n}, \tau\right), U_{-}\left(t_{n}, \tau\right)\right)=J \mathcal{E}\left(t_{n}, \tau, U_{+}\left(t_{n}, \tau\right)+\mathrm{e}^{\tau J} U_{-}\left(t_{n}, \tau\right)\right), \\
& \mathcal{F}_{-}\left(t_{n}, \tau, U_{+}\left(t_{n}, \tau\right), U_{-}\left(t_{n}, \tau\right)\right)=-J \mathrm{e}^{-\tau J} \mathcal{E}\left(t_{n}, \tau, U_{+}\left(t_{n}, \tau\right)+\mathrm{e}^{\tau J} U_{-}\left(t_{n}, \tau\right)\right) .
\end{aligned}
$$

In all, the detailed numerical scheme of the solver towards $(2.11)$ reads as follows. Denote $\left(U_{ \pm}\right)^{n}(\tau) \approx$ $U_{ \pm}\left(t_{n}, \tau\right)$ for $n \geq 0$ and let $U_{ \pm}^{0}=U_{ \pm}(0, \tau)$ the initial condition given by (2.26). We update the $U_{ \pm}^{n}$ for $n \geq 1$ as

$$
\begin{aligned}
& {\widehat{\left(U_{ \pm}\right)_{\ell}}}_{\ell}^{1}=\mathrm{e}^{-\frac{i \ell \Delta t}{\varepsilon^{2}}}{\widehat{\left(U_{ \pm}\right)_{\ell}}}_{\ell}^{0}+p_{\ell}{\widehat{\left(\mathcal{F}_{ \pm}\right)_{\ell}}}_{\ell}^{0}+q_{\ell} \frac{1}{\Delta t}\left({\widehat{\left(\mathcal{F}_{ \pm}\right)_{\ell}}}_{\ell}^{* 1}-{\widehat{\left(\mathcal{F}_{ \pm}\right)_{\ell}^{0}}}^{0}\right. \\
& {\widehat{\left(U_{ \pm}\right)_{\ell}}}_{\ell}^{n+1}=\mathrm{e}^{-\frac{i \ell \Delta t}{\varepsilon^{2}}}{\widehat{\left(U_{ \pm}\right)_{\ell}^{n}}}^{n}+p_{\ell}{\widehat{\left(\mathcal{F}_{ \pm}\right)_{\ell}}}_{\ell}^{n}+q_{\ell} \frac{1}{\Delta t}\left({\widehat{\left(\mathcal{F}_{ \pm}\right)_{\ell}}}_{\ell}^{n}-{\widehat{\left(\mathcal{F}_{ \pm}\right)_{\ell}}}_{\ell}^{n-1}\right), \quad \forall n \geq 1,
\end{aligned}
$$

where

$$
U_{ \pm}^{n}(\tau)=\sum_{\ell=-N_{\tau} / 2}^{N_{\tau} / 2-1}{\widehat{\left(U_{ \pm}\right)_{\ell}}}^{n} \mathrm{e}^{i \ell \tau}, \quad \mathcal{F}_{ \pm}^{n}(\tau)=\sum_{\ell=-N_{\tau} / 2}^{N_{\tau} / 2-1}{\widehat{\left(\mathcal{F}_{ \pm}\right)_{\ell}}}^{n} \mathrm{e}^{i \ell \tau}, \quad \mathcal{F}_{ \pm}^{*, 1}(\tau)=\sum_{\ell=-N_{\tau} / 2}^{N_{\tau} / 2-1}{\widehat{\left(\mathcal{F}_{ \pm}\right)}}_{\ell}^{*, 1} \mathrm{e}^{i \ell \tau}, \quad n \geq 0
$$

and

$$
\begin{aligned}
& \mathcal{F}_{+}^{n}(\tau)=J \mathcal{E}^{n}\left(\tau, U_{+}^{n}(\tau)+\mathrm{e}^{\tau J} U_{-}^{n}(\tau)\right), \mathcal{F}_{-}^{n}(\tau):=-J \mathrm{e}^{-\tau J} \mathcal{E}^{n}\left(\tau, U_{+}^{n}(\tau)+\mathrm{e}^{\tau J} U_{-}^{n}(\tau)\right), n \geq 0, \\
& \mathcal{F}_{+}^{*, 1}(\tau)=J \mathcal{E}^{*, 1}\left(\tau, U_{+}^{*, 1}(\tau)+\mathrm{e}^{\tau J} U_{-}^{*, 1}(\tau)\right), \mathcal{F}_{-}^{*, 1}(\tau):=-J \mathrm{e}^{-\tau J} \mathcal{E}^{1, *}\left(\tau, U_{+}^{*, 1}(\tau)+\mathrm{e}^{\tau J} U_{-}^{*, 1}(\tau)\right),
\end{aligned}
$$

with

$$
{\widehat{\left(U_{ \pm}\right)_{\ell}}}_{\ell}^{*, 1}=\mathrm{e}^{-\frac{i \ell \Delta t}{\varepsilon^{2}}}{\widehat{\left(U_{ \pm}\right)_{\ell}}}^{0}+p_{\ell}{\widehat{\left(\mathcal{F}_{ \pm}\right)_{\ell}}}^{0}, \quad U_{ \pm}^{*, 1}(\tau)=\sum_{\ell=-N_{\tau} / 2}^{N_{\tau} / 2-1}{\widehat{\left(U_{ \pm}\right)_{\ell}}}^{*, 1} \mathrm{e}^{i \ell \tau}
$$


The numerical electrical field $\mathcal{E}^{n}$ is given by collecting all the two-scale characteristics $\left(U_{ \pm}^{n}\right)_{k}, k=1, \ldots, N_{p}$ and solving the Poisson equation:

$$
\begin{aligned}
& \nabla_{\mathbf{x}} \cdot \mathcal{E}^{n}(\tau, \mathbf{x})=\sum_{k=1}^{N_{p}} \omega_{k} S\left(\mathbf{x}-X_{k}^{n}(\tau)\right)-n_{i}, \quad X_{k}^{n}(\tau)=U_{+, k}^{n}(\tau)+\mathrm{e}^{\tau J} U_{-, k}^{n}(\tau) \\
& \nabla_{\mathbf{x}} \cdot \mathcal{E}^{*, 1}(\tau, \mathbf{x})=\sum_{k=1}^{N_{p}} \omega_{k} S\left(\mathbf{x}-X_{k}^{*}(\tau)\right)-n_{i}, \quad X_{k}^{*}(\tau)=U_{+, k}^{*, 1}(\tau)+\mathrm{e}^{\tau J} U_{-, k}^{*, 1}(\tau),
\end{aligned}
$$

where $S(\mathbf{x})$ is the regularised basis (the choice of quintic splines has been done in our computations).

We conclude this section with the following proposition which states that the so-obtained numerical scheme is uniformly accurate with respect to $\varepsilon$.

Proposition 2.2. Let $U_{ \pm}$the solution of $(2.11)$ on $[0, T] \times[0,2 \pi]$ subject to the initial condition (2.26). Let $\left(U_{ \pm}^{n}\right), n \geq 0$ defined by the numerical scheme (2.30) with the initial data (2.26). Then, the following estimate holds

$$
\sup _{\varepsilon \in] 0,1]}\left\|U_{ \pm}\left(t_{n}, \cdot\right)-U_{ \pm}^{n}(\cdot)\right\|_{L_{\tau}^{\infty}} \leq C \Delta t^{2}
$$

with $C>0$, for all $n=0, \ldots, N$ with $N \Delta t=T$ is the final time.

Proof. By Taylor expansion (with integral remainder) of $\left.V\left(t_{n}+s\right):=\widehat{(\mathcal{F}}_{ \pm}\right)_{\ell}\left(t_{n}+s\right)$ in $(2.29)$, we get

$$
\begin{aligned}
V\left(t_{n}+s\right)= & V\left(t_{n}\right)+\frac{s}{\Delta t}\left[V\left(t_{n}\right)-V\left(t_{n-1}\right)\right] \\
& +\int_{0}^{1}(1-t) s^{2} V^{\prime \prime}\left(t_{n}+s t\right) d t+s \int_{0}^{1}(1-t) \Delta t V^{\prime \prime}\left(t_{n}-t \Delta t\right) d t .
\end{aligned}
$$

Then, the exact solution $\widehat{\left(U_{ \pm}\right)}(t)$ satisfies the scheme $(2.30)$ up to the following remainder term $\mathcal{R}$

$$
\begin{aligned}
\mathcal{R}:= & \int_{0}^{\Delta t} \mathrm{e}^{-\frac{i \ell}{\varepsilon^{2}}(\Delta t-s)} s^{2} \int_{0}^{1}(1-t) V^{\prime \prime}\left(t_{n}+s t\right) d t d s \\
& +\int_{0}^{\Delta t} \mathrm{e}^{-\frac{i \ell}{\varepsilon^{2}}(\Delta t-s)} s \int_{0}^{1} \Delta t(1-t) V^{\prime \prime}\left(t_{n}-t \Delta t\right) d t d s .
\end{aligned}
$$

Thanks to Proposition 2.1, $\left\|\partial_{t}^{2} U\right\|_{L_{t, \tau}^{\infty}}$ is uniformly bounded (the derivatives of $\mathcal{F}$ are bounded, as in [3]) so that

$$
|\mathcal{R}| \leq\left(\int_{0}^{\Delta t} s^{2} d s+\Delta t \int_{0}^{\Delta t} s d s\right)\left\|V^{\prime \prime}\right\|_{L_{t, \tau}^{\infty}} \leq C \Delta t^{3}
$$

Now, we define the error $\mathcal{E}^{n}:=\left|{\widehat{\left(U_{ \pm}\right)}}\left(t_{n}\right)-{\widehat{\left(U_{ \pm}\right)}}^{n}\right|$, which satisfies the following estimate

$$
\mathcal{E}^{n+1} \leq \mathcal{E}^{n}+C \int_{0}^{\Delta t}\left(\mathcal{E}^{n}+\mathcal{E}^{n-1}\right) d s+C \Delta t^{3} \leq \mathcal{E}^{n}(1+C \Delta t)+C \Delta t \mathcal{E}^{n-1}+C \Delta t^{3}
$$

We proceed by induction to prove there exisits $C>0$ such that $\mathcal{E}^{n} \leq C \Delta t^{2}(1+C \Delta t)^{n}, \forall n \geq 1$. For the first iteration, from $\mathcal{E}^{0}=0$ then we get $\mathcal{E}^{1} \leq C \Delta t^{2}$ (since a prediction-correction scheme is used for the first iteration). Thanks to this estimate, we conclude the proof using the following

$$
\mathcal{E}^{n} \leq C \Delta t^{2}(1+C \Delta t)^{n} \leq C \Delta t^{2} \mathrm{e}^{C n \Delta t} \leq C \Delta t^{2} \mathrm{e}^{C T}
$$

where $T=N \Delta t$ is the final time. 
The proposed exponential integrator (2.30)-(2.31) is self-consistent for solving (2.11). During the computation, the scheme runs with $U_{ \pm}^{n}(\tau)$ only. It does not require approximations of the original components $\mathbf{x}(t), \mathbf{y}(t)$ for $(2.6)$ or $X(t, \tau), Y(t, \tau)$ for $(2.7)$. In addition to the second order uniform accuracy in time and the spectral accuracy in $\tau$, the scheme (2.30)-(2.31) is fully explicit and efficient thanks to fast Fourier transform in $\tau$ and the locality of the regularised basis $S(\mathbf{x})$.

With $\left(U_{ \pm}^{n}\right)_{k}, k=1, \ldots, N_{p}$ from the exponential integrator (2.30)-(2.31), one can obtain the approximation of the solution of the original characteristics (2.2) as

$$
\mathbf{x}_{k}\left(t_{n}\right) \approx\left(U_{+}^{n}\left(t_{n} / \varepsilon^{2}\right)+e^{J t_{n} / \varepsilon^{2}} U_{-}^{n}\left(t_{n} / \varepsilon^{2}\right)\right)_{k}=X_{k}^{n}\left(\frac{t_{n}}{\varepsilon^{2}}\right), \quad \mathbf{v}_{k}\left(t_{n}\right) \approx \frac{J}{\varepsilon} \mathrm{e}^{J \frac{t_{n}}{\varepsilon^{2}}} U_{-, k}^{n}\left(\frac{t_{n}}{\varepsilon^{2}}\right), \quad n \geq 1 .
$$

Then together with the PIC approximation (2.1), we complete the numerical scheme of a two-scale PIC method for solving the Vlasov-Poisson equation (1.1).

\section{Numerical results}

In this section, we first test the accuracy of the proposed UA PIC method (2.30)-(2.31) and then conduct some numerical experiments to explore the dynamics of the Vlasov-Poisson equation (1.1) in the limit regime.

\subsection{Example 1}

We consider the Vlasov-Poisson equation (1.1) with the Kelvin-Helmholtz instability type initial data $[7,21,12]$,

$$
f_{0}(\mathbf{x}, \mathbf{v})=\frac{1}{2 \pi}\left(1+\sin \left(x_{2}\right)+\eta \cos \left(k x_{1}\right)\right) \mathrm{e}^{-\frac{|\mathbf{v}|^{2}}{2}}
$$

on a computational domain for $\mathbf{x}=\left(x_{1}, x_{2}\right)$ as $\Omega=[0,2 \pi / k] \times[0,2 \pi]$ for some $k, \eta>0$. Periodic boundary conditions are assumed in $\mathbf{x}$.

Accuracy test. In order to show the importance of the preparation of the initial data for the UA property, we consider the following initial data for each two-scale characteristic (2.11):

- $U_{ \pm}(0, \tau)=\mathbf{u}_{ \pm}^{0}$ (we will refer as $\left.U^{0 t h}\right)$;

- $U_{ \pm}(0, \tau)=U_{ \pm}^{1 s t}$; here the electric field $\mathcal{E}^{1 s t}$ is replaced in the Chapman-Enskog expansion $(2.26)$ by the initial electric field $E(t=0, \mathbf{x})$ solution of $\nabla_{\mathbf{x}} \cdot E(t=0, \mathbf{x})=\sum_{\ell=1}^{N_{p}} \omega_{\ell} \delta\left(\mathbf{x}-\mathbf{x}_{\ell, 0}\right)$;

- $U_{ \pm}(0, \tau)=U_{ \pm}^{2 n d}$ given by $(2.26)$.

We shall test the performance of the proposed scheme (2.30)-(2.31) under the two inefficient choices of initial data $U_{ \pm}^{0 t h}$ and $U_{ \pm}^{1 s t}$. We shall compare the results with the one obtained from the two-scale PIC (2.30)-(2.31) under the well-prepared initial data $U_{ \pm}^{2 n d}(2.26)$.

We choose $\eta=0.05, k=0.5$ in (2.26) for this accuracy test. We discretize the spatial domain $\Omega$ with 64 points in $x_{1}$-direction and 32 points in $x_{2}$-direction. The Poisson equation $(1.1 \mathrm{~b})$ is solved on $\Omega$ with periodic boundary conditions by the fast Fourier transform where the background density $n_{i}$ is such that the average in space of total charge vanishes. We take the basis function $S(\mathbf{x})$ for the PIC method as the fifth order B-spline (same in other numerical experiments), i.e. $m=5$ in (2.4). The high order spline function is chosen here to reach a high accuracy in spatial approximation so that the temporal error can be distinguished. We choose the numerical grid points in $\tau$-direction as $N_{\tau}=32$. We take $N_{p}=204800$ and generate the initial position and velocity $\left(\mathbf{x}_{k, 0}, \mathbf{v}_{k, 0}\right)$ of each particle by means of the rejection sampling.

We compute the error on the density function $\rho^{\varepsilon}(t, \mathbf{x})=\int_{\mathbb{R}^{2}} f^{\varepsilon}(t, \mathbf{x}, \mathbf{v}) d \mathbf{v}, \mathbf{x} \in \Omega$ at the final time $t=1$ where a reference solution is obtained numerically via the UA PIC with very small time step, e.g. $\Delta t=10^{-3}$. We measure the error under maximum norm in space. The temporal error test results of the proposed twoscale PIC method (2.30)-(2.31) with the three types of initial data $U_{ \pm}^{0 t h}, U_{ \pm}^{1 s t}, U_{ \pm}^{2 n d}$ are given in Figure 1. We can observe the influence of the initial data on the convergence of the method. When the initial data 

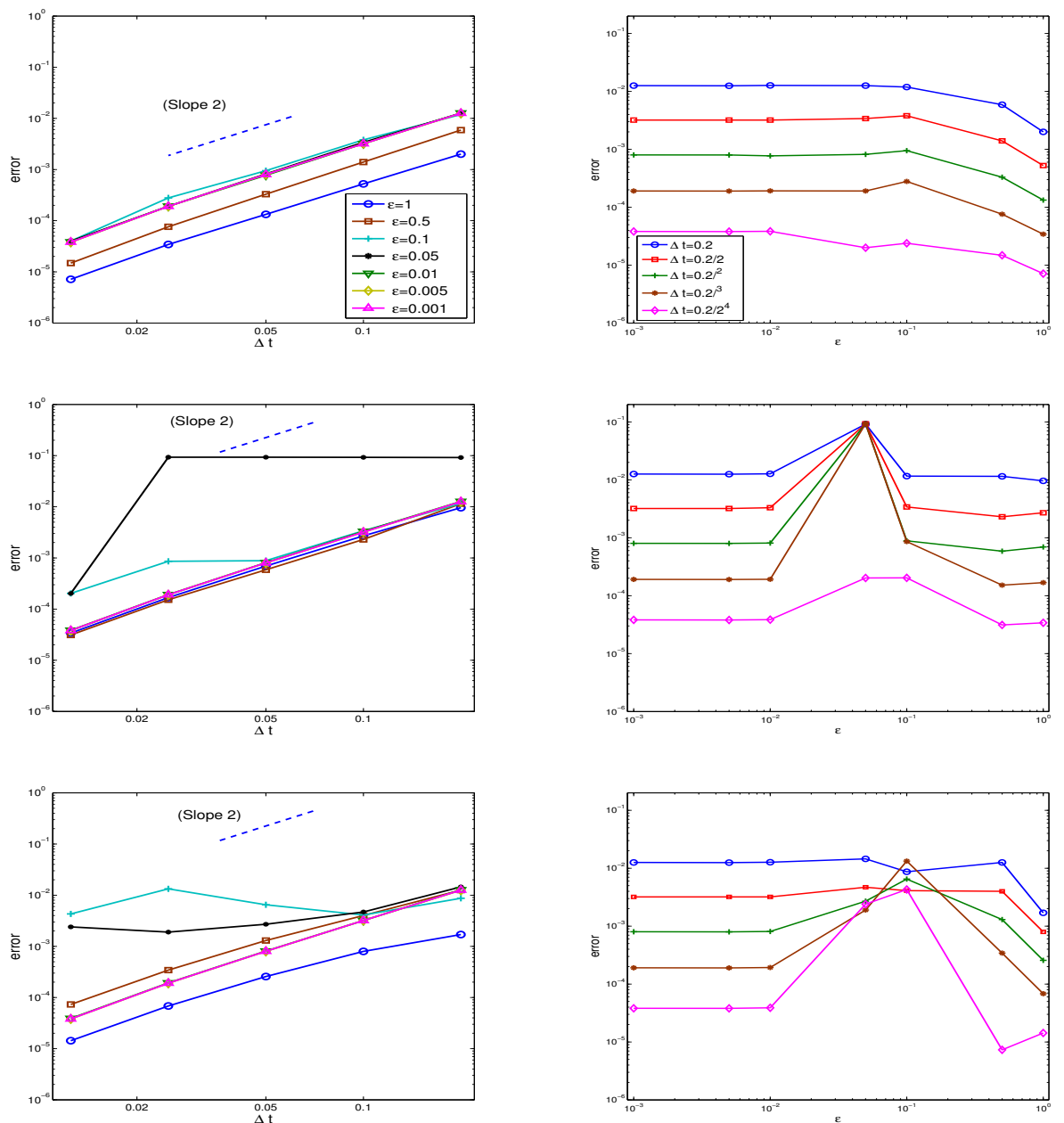

Figure 1: Temporal error of the proposed PIC method in $\rho^{\varepsilon}$ with respect to $\Delta t$ and $\varepsilon$ : results of $U_{ \pm}^{2 n d}$ (first row); results of $U_{ \pm}^{1 s t}$ (second row); results of $U_{ \pm}^{0 t h}$ (third row).

is chosen as $U_{ \pm}^{2 n d}$, the uniform accuracy is well recovered whereas order reduction (or even no convergence) can be observed when the initial data is not well-prepared, in particular in the intermediate regime. We also study the numerical error to further address the error from the velocity part, considering the following quantities

$$
\rho_{\mathbf{v}, 1}^{\varepsilon}(t, \mathbf{x}):=\int_{\mathbb{R}^{2}}\left(\left|v_{1}\right|+\left|v_{2}\right|\right) f^{\varepsilon}(t, \mathbf{x}, \mathbf{v}) d \mathbf{v}, \quad \rho_{\mathbf{v}, 2}^{\varepsilon}(t, \mathbf{x}):=\int_{\mathbb{R}^{2}}|\mathbf{v}|^{2} f^{\varepsilon}(t, \mathbf{x}, \mathbf{v}) d \mathbf{v}, \quad \mathbf{x} \in \Omega
$$

The errors of the UA PIC regarding $\rho_{\mathbf{v}, 1}^{\varepsilon}$ and $\rho_{\mathbf{v}, 2}^{\varepsilon}$ at $t=1$ are shown in Figure 2, with the initial condition $U_{ \pm}^{2 n d}$ (under the similar setup as previously). The uniform accuracy is still observed on these quantities. Then, in the following, UA PIC scheme will always refer to the scheme (2.30)-(2.31) with the initial data $U_{ \pm}^{2 n d}$.

Figure 3 investigates the error in $\tau$-direction (using the maximum norm) by comparing the solution obtained by the UA PIC scheme under $\Delta t=10^{-3}$ and different $N_{\tau}$ to a reference one obtained under $N_{\tau}=32$ (the other numerical parameters are the same as previously). Figure 3 illustrates the spectral accuracy of the scheme in the $\tau$-direction, meaning that a few number of grid points are sufficient to obtain 

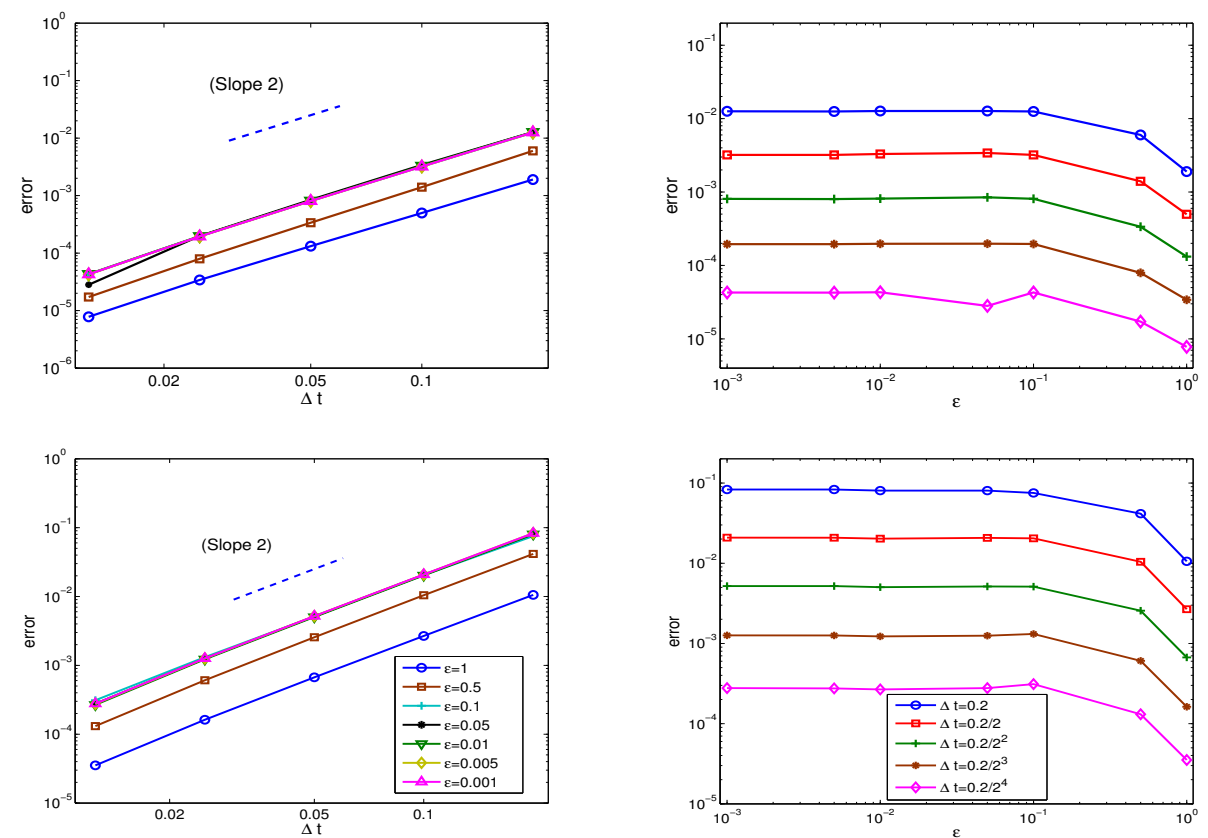

Figure 2: Temporal error of the proposed PIC method under $U_{ \pm}^{2 n d}$ : results in $\rho_{\mathbf{v}, 1}^{\varepsilon}$ (first row) and results in $\rho_{\mathbf{v}, 2}^{\varepsilon}$ (second row) with respect to $\Delta t$ and $\varepsilon$.

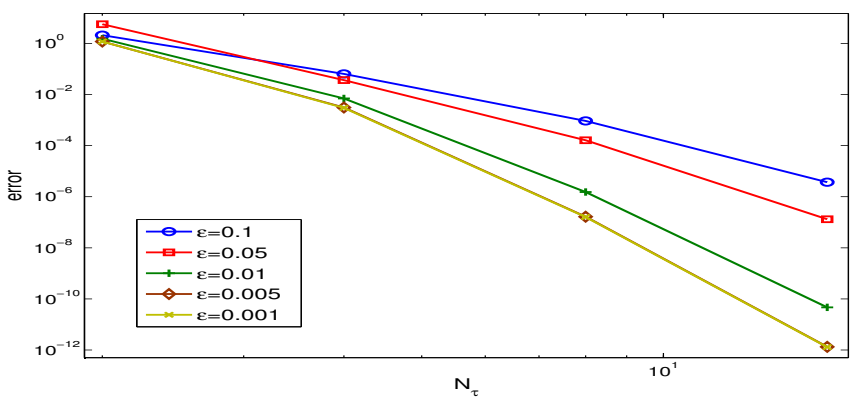

Figure 3: Error of with respect to $N_{\tau}$ under different $\varepsilon$.

a good accuracy.

Finally, the time history of the relative total energy $(H(t)-H(0)) / H(0)$ is displayed in Figure 4 , where $H(t)$ is a conserved quantity defined by

$$
H(t)=\frac{1}{2} \int_{\mathbb{R}^{2}} \int_{\Omega}|\mathbf{v}|^{2} f^{\varepsilon}(t, \mathbf{x}, \mathbf{v}) d \mathbf{x} d \mathbf{v}+\frac{1}{2} \int_{\Omega}\left|E^{\varepsilon}(t, \mathbf{x})\right|^{2} d \mathbf{x} .
$$

The error of the total energy, i.e. $\left|H\left(t_{n}\right)-H(0)\right| / H(0)$ during the computation of the UA PIC (i.e. with $U_{ \pm}^{2 n d}$ ) is given in Figure 4 under time step $\Delta t=0.1$ and $\Delta t=0.05$ for several $\varepsilon$. We can observe that the error is well preserved and in particular, its amplitude is divided by 4 when the time step is divided by 2 , as expected.

Numerical exploration. Next, we carry out some numerical explorations on the Vlasov-Poisson equation (1.1). We firstly investigate numerically the convergence of the Vlasov-Poisson equation (1.1) to its limit 

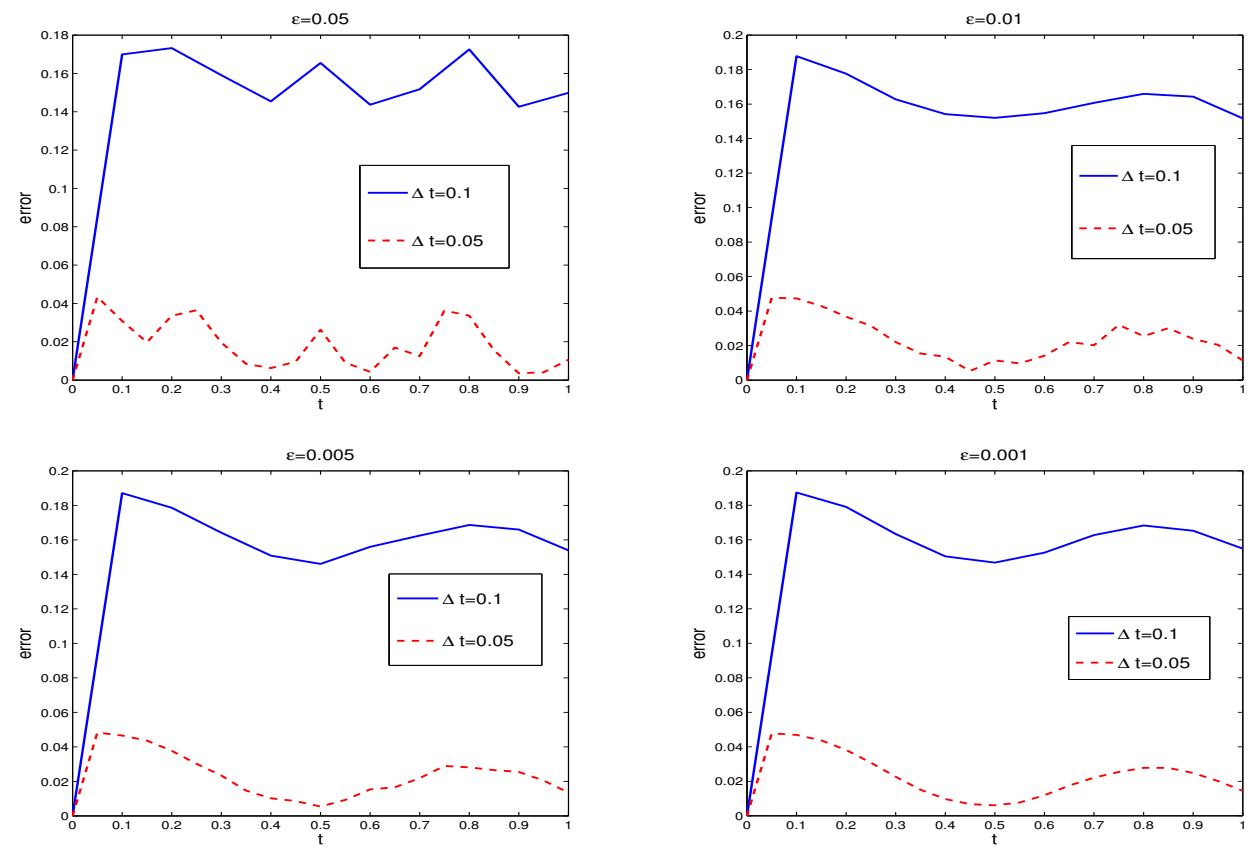

Figure 4: Time history of the relative error of the total energy for the UA PIC scheme, with $\Delta t=0.05,0.1$.

model (1.2). We work under the initial data (3.1) with $\eta=0.05, k=0.5$. We solve the asymptotic model (1.2) satisfied by $f(t, \mathbf{x}, \mathbf{v})$ using a standard PIC method (a leap-frog scheme is used for the characteristics). We study the following quantities

$$
\begin{aligned}
\rho^{\varepsilon}(t, \mathbf{x})-\rho(t, \mathbf{x}) & :=\int_{\mathbb{R}^{2}} f^{\varepsilon}(t, \mathbf{x}, \mathbf{v}) d \mathbf{v}-\int_{\mathbb{R}^{2}} f(t, \mathbf{x}, \mathbf{v}) d \mathbf{v}, \quad \mathbf{x} \in \Omega \\
\rho_{\mathbf{v}, 2}^{\varepsilon}(t, \mathbf{x})-\rho_{\mathbf{v}, 2}(t, \mathbf{x}) & :=\int_{\mathbb{R}^{2}}|\mathbf{v}|^{2} f^{\varepsilon}(t, \mathbf{x}, \mathbf{v}) d \mathbf{v}-\int_{\mathbb{R}^{2}}|\mathbf{v}|^{2} f(t, \mathbf{x}, \mathbf{v}) d \mathbf{v}, \quad \mathbf{x} \in \Omega
\end{aligned}
$$

in the $L^{\infty}$ norm in space, for different values of $\varepsilon$, at time $t=1$. In Figure 5 , these errors are displayed as a function of $\varepsilon$. In both cases, a first order in $\varepsilon$ is obtained. These results confirm the strong convergence of Vlasov-Poisson equation (1.1) towards the asymptotic model (1.2).
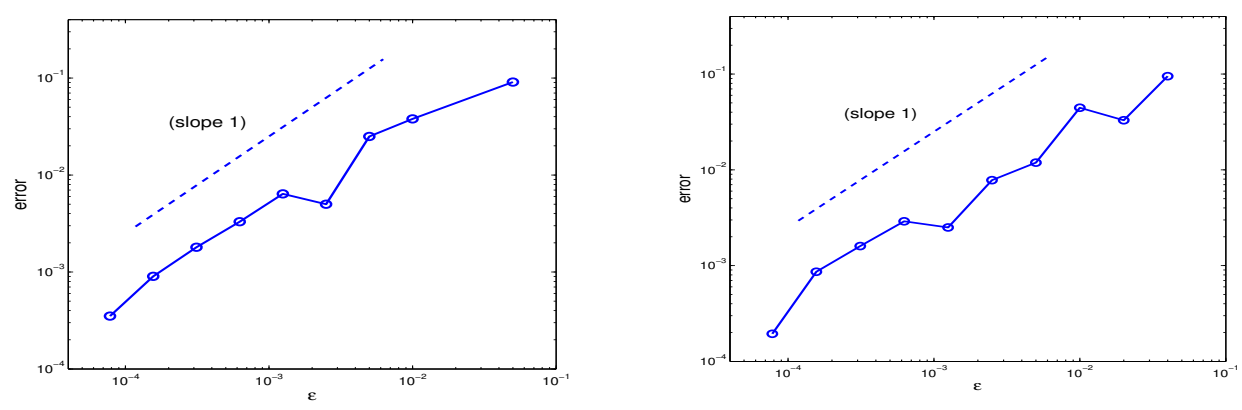

Figure 5: Convergence of the Vlasov-Poisson equation (1.1) towards the asymptotic model $(1.2):\left\|\rho^{\varepsilon}(t=1, \cdot)-\rho(t=1, \cdot)\right\|_{L^{\infty}}$ (left) and $\left\|\rho_{\mathbf{v}}^{\varepsilon}(t=1, \cdot)-\rho_{\mathbf{v}}(t=1, \cdot)\right\|_{L^{\infty}}$ (right) as a function of $\varepsilon$. 
Then, we consider the Root Mean Square (RMS) quantity in this example:

$$
R M S(t):=\sqrt{\int_{\Omega} x_{1}^{2}\left(\rho^{\varepsilon}(t, \mathbf{x})-n_{i}\right) d \mathbf{x},} \quad t \geq 0,
$$

which fast oscillates in time with frequency $O\left(\varepsilon^{-2}\right)$ as $0<\varepsilon \ll 1$. We consider this quantity using the UA PIC scheme with the initial data $U_{+}^{2 n d}$, for $\varepsilon=0.025$ and $\varepsilon=0.0125$. On Figure 6 , we plot the time history of $R M S$ using the UA PIC scheme under a comparatively large time step $\Delta t=0.025,0.05,0.1$, together with a reference solution obtained with a resolved time step. From the results, we can see that the UA PIC scheme is able to capture the correct RMS quantity at different time even though the time step is too large to resolve the oscillation waves (the ratio $\Delta t / \varepsilon^{2}$ takes values up to 640 ).
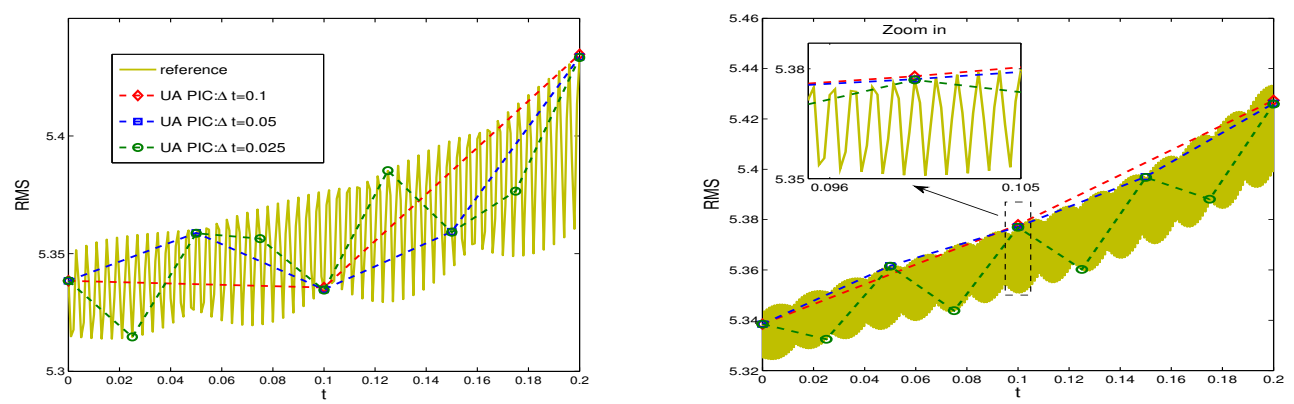

Figure 6: Time history of $R M S(t)$ for $\varepsilon=0.025$ (left) and $\varepsilon=0.0125$ (right) by using UA PIC with $\Delta t=0.025,0.05,0.1$.
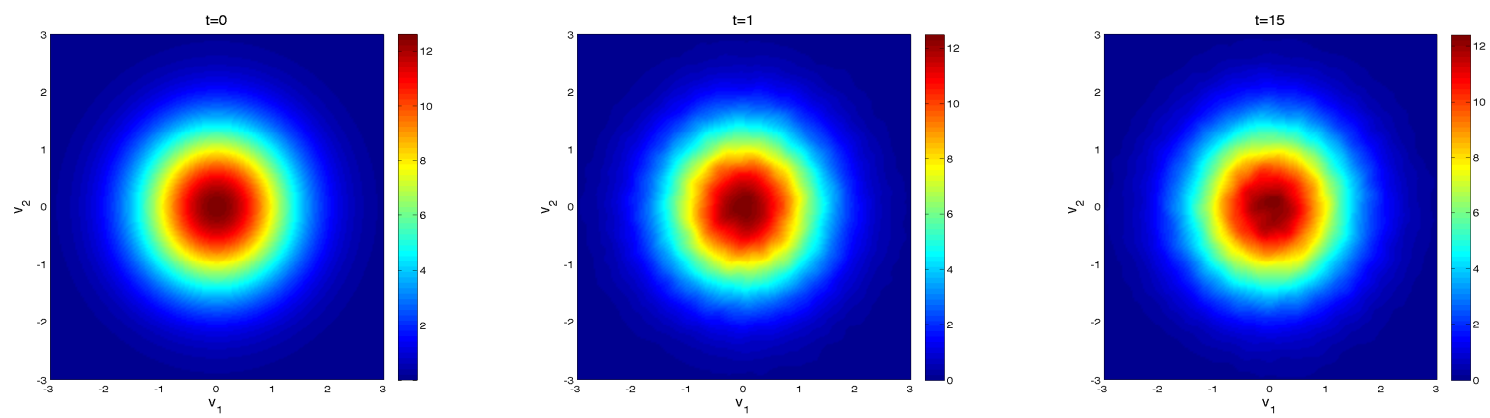

Figure 7: Contour plot of quantity $\chi^{\varepsilon}(t, \mathbf{v})$ at different $t$ with $\varepsilon=0.005$.

Finally, we perform simulations for a larger final time. To validate our simulations, we consider the asymptotic regime $\varepsilon=0.005$ so that $f^{\varepsilon}$ is close to $f$ solution of (1.2). Using an isotropic (in $\mathbf{v}$ ) initial condition (3.1) implies that the solution $f$ remains isotropic for all time. Then, the asymptotic model (1.2) can be integrated with respect to $\mathbf{v}$ to get the guiding-center model (1.3). For this model, the linear theory enables to determine a priori an instability rate for the initially excited modes of the electric potential $([7,12])$. This rate can be compared to our numerical results obtained by the UA PIC solver when $\varepsilon$ is small. Then, with $\varepsilon=0.005$, we consider the dynamics of the following quantity

$$
\xi(t):=\ln \left(\left|\widehat{\left(\phi_{1,1}^{\varepsilon}\right)}(t)\right|\right), \quad t \geq 0
$$


where $\widehat{\left(\phi_{1,1}^{\varepsilon}\right)}$ denotes the Fourier coefficient of $\phi^{\varepsilon}$ of mode $(1,1)$. Figure 8 shows the time history of $\xi(t)$ under different values of $\eta$ and $k$. We observe that when $\eta$ is small, the linear phase is larger, and for a given $k$, the numerical rate is in a very good agreement with the analytical one. In Figure 9 (resp. Figure 7), we
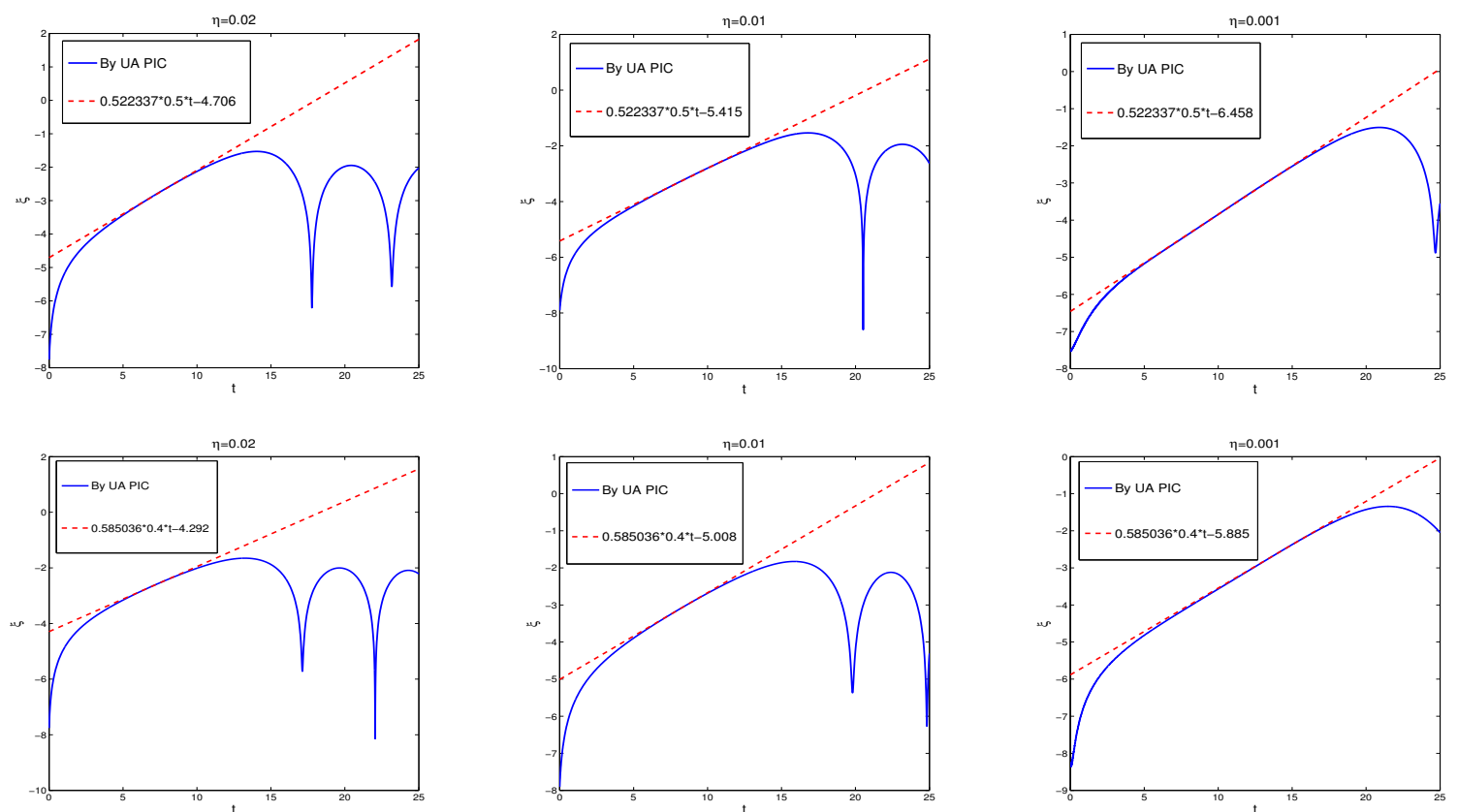

Figure 8: Time history of the quantity $\xi(t)$ (3.3) under different $\eta$ with $\varepsilon=0.005, k=0.5$ (first row) and $k=0.4$ (second row).

study the space (resp. velocity) profiles $\rho^{\varepsilon}(t, \mathbf{x})-n_{i}\left(\operatorname{resp} . \chi^{\varepsilon}(t, \mathbf{v}):=\int_{\Omega} f^{\varepsilon}(t, \mathbf{x}, \mathbf{v}) d \mathbf{x}\right)$, at different times. The evolution of $\rho^{\varepsilon}(t, \mathbf{x})-n_{i}$ is in very good agreement with the results obtained in the literature [7, 12]. We can remark in Figure 7 that there is no dynamics in the velocity direction. As mentioned above, this is explained by the isotropic character of the initial condition (3.1).

\subsection{Example 2}

Then, we apply the UA PIC scheme to study the Vlasov-Poisson equation (1.1) with a non-isotropic (in v) initial data:

$$
f_{0}(\mathbf{x}, \mathbf{v})=\frac{1}{4 \pi}\left(1+\sin \left(x_{2}\right)+\eta \cos \left(k x_{1}\right)\right)\left(\mathrm{e}^{-\frac{\left(v_{1}+2\right)^{2}+v_{2}^{2}}{2}}+\mathrm{e}^{-\frac{\left(v_{1}-2\right)^{2}+v_{2}^{2}}{2}}\right) .
$$

As discussed previously, since the initial data is no more isotropic and at least when $\varepsilon<<1$, a non trivial dynamics should be observed in the velocity direction. We choose $\eta=0.05, k=0.5$ and take $N_{p}=$ $409600, N_{\tau}=16$. We compute the solution under $\varepsilon=0.005$ at different times. The dynamics of $\chi^{\varepsilon}(t, \mathbf{v}):=$ $\int_{\Omega} f^{\varepsilon}(t, \mathbf{x}, \mathbf{v}) d \mathbf{x}$ is shown in Figure 10. The profiles of $\rho^{\varepsilon}$ are similar to the results in (9), so we omit them for brevity. For small times, the two bumps of $\chi^{\varepsilon}$ start to rotate, at $t \approx 15$, the bumps merge and for large times, the velocity distribution becomes isotropic. These effects can not be observed using the guiding-center model (1.3) since the associated initial condition is the integral with respect to $\mathbf{v}$ of (3.4).

\subsection{Long time test}

At last but no least, we study the behavior of the UA PIC scheme with the initial condition (3.1) and for a large final time $\left(t_{f}=100\right)$. We fix the numerical parameters as follows: $\Delta t=0.1, N_{x}=128, N_{y}=$ 

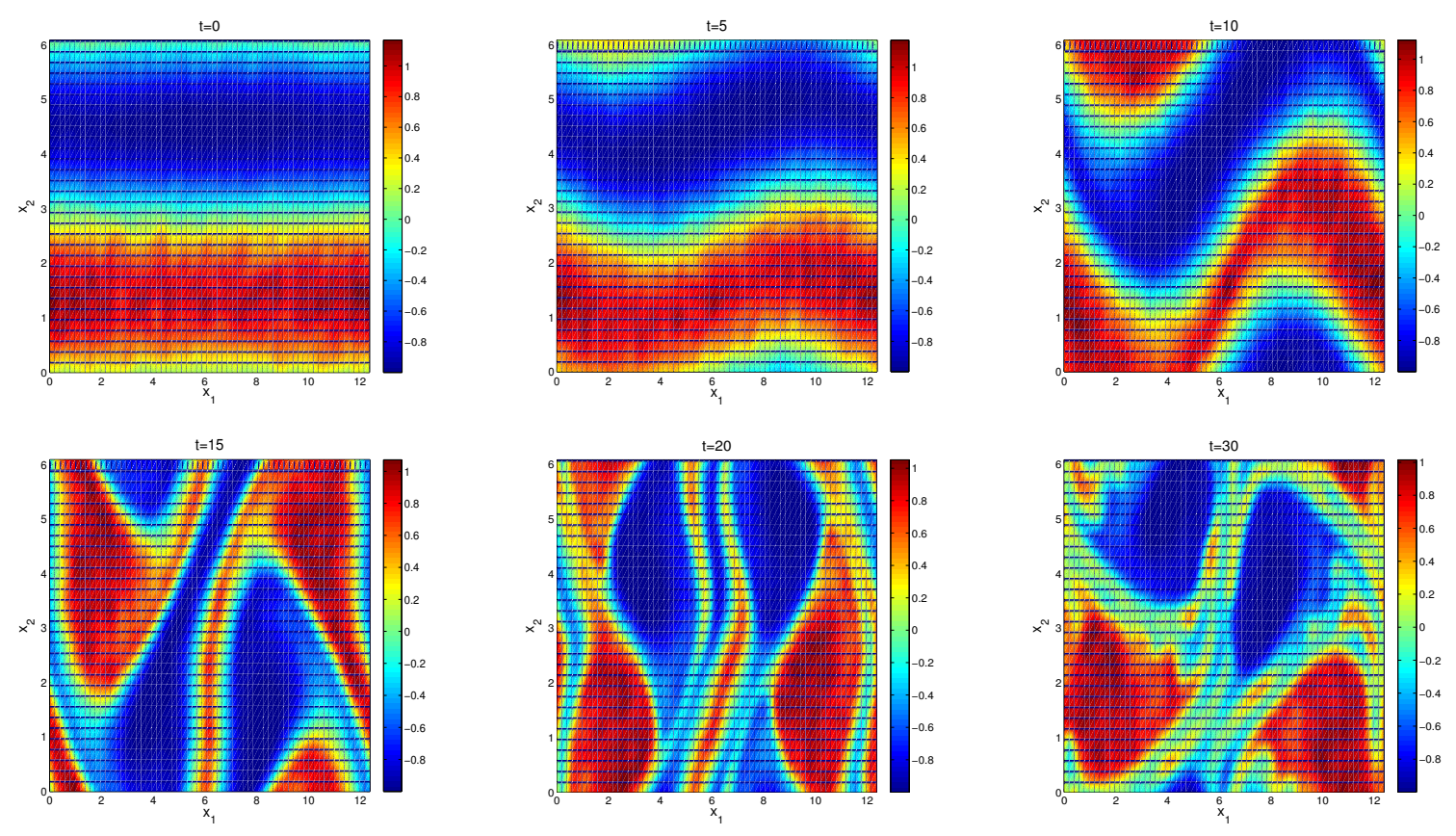

Figure 9: Contour plot of quantity $\rho^{\varepsilon}(t, \mathbf{x})-n_{i}$ at different $t$ with $\varepsilon=0.005$.
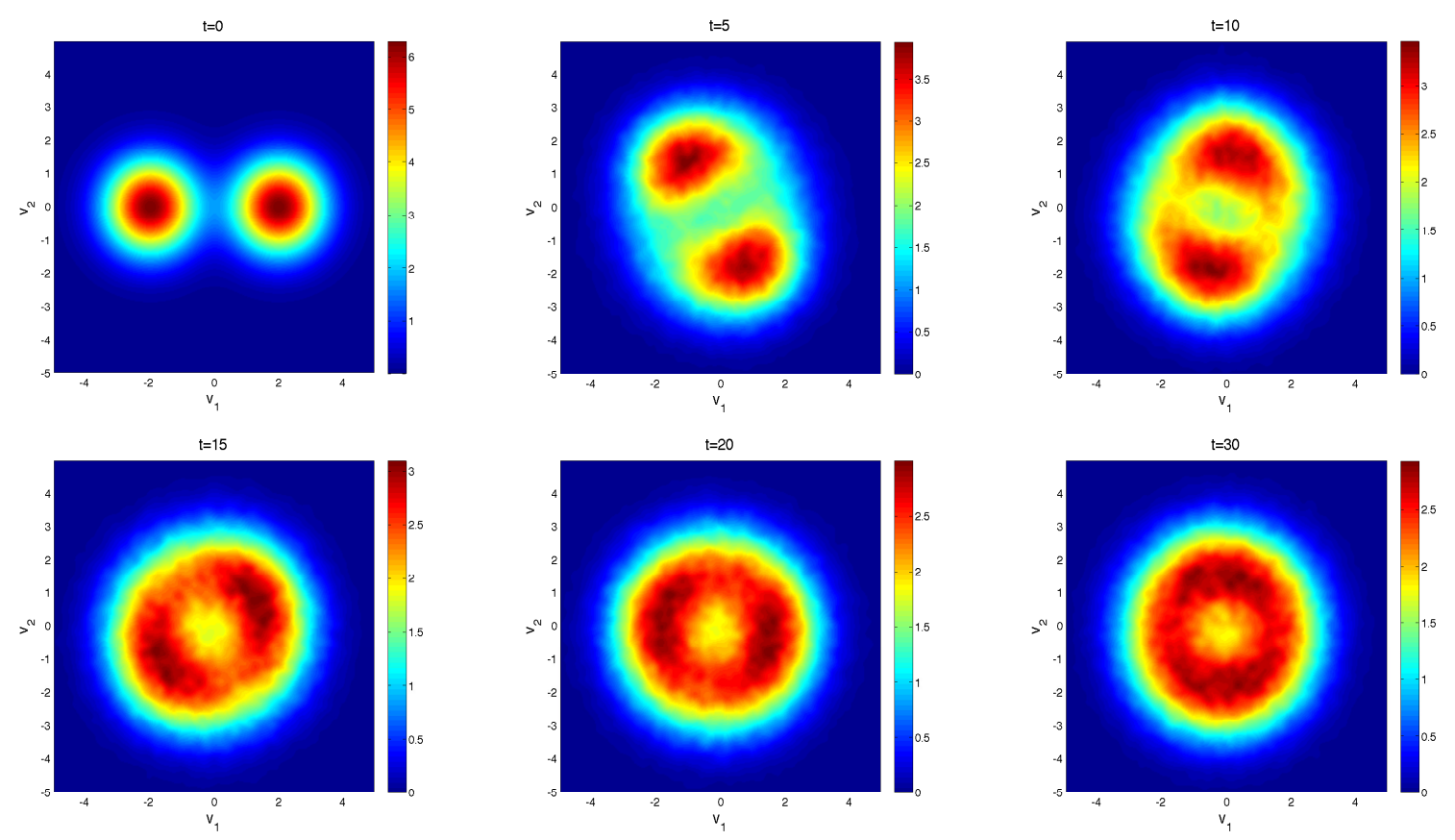

Figure 10: Contour plot of quantity $\chi^{\varepsilon}(t, \mathbf{v})$ at different $t$ with $\varepsilon=0.005$. 

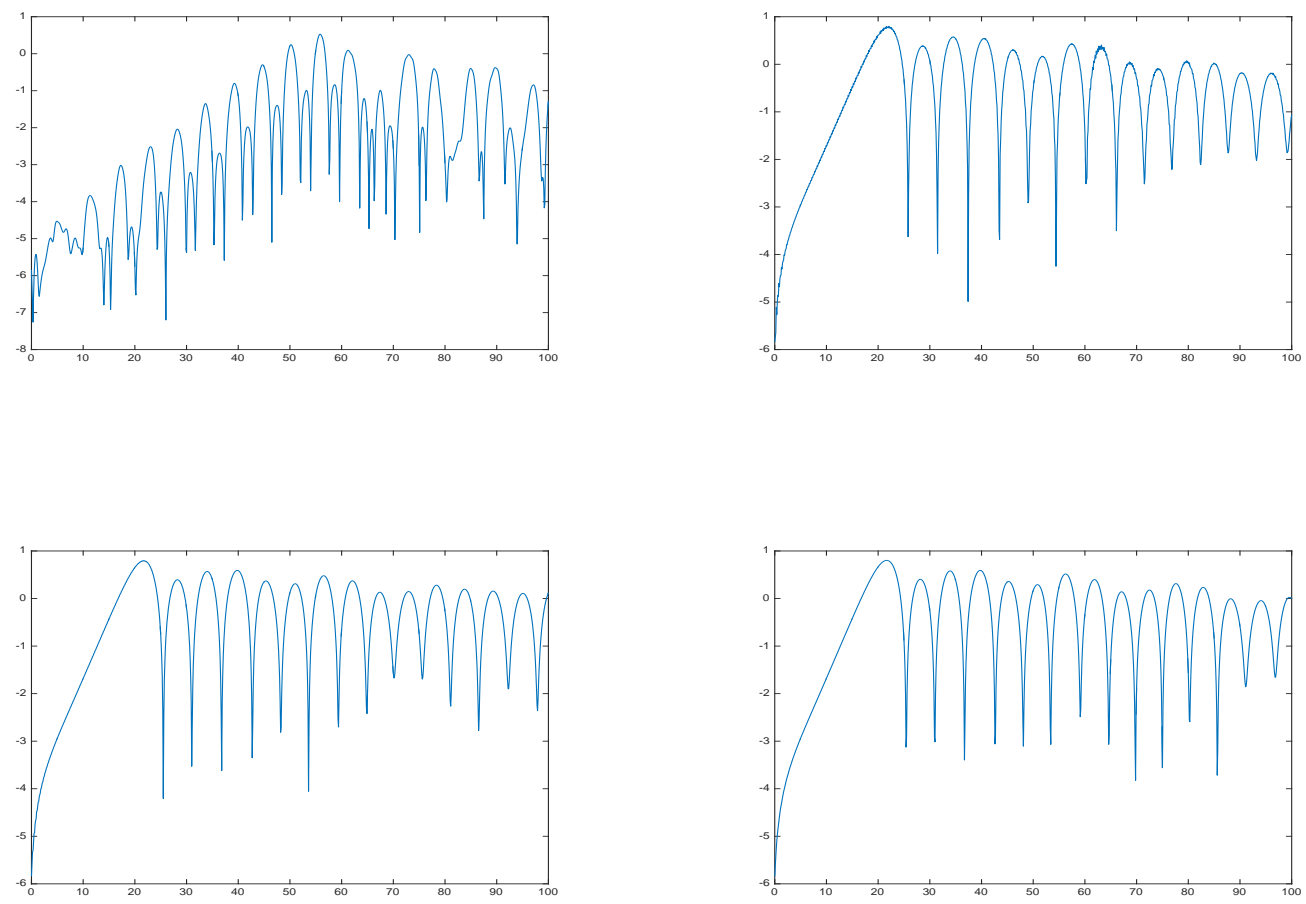

Figure 11: Time history the quantity $\xi(t)$ (3.3) for different values of $\varepsilon=1,0.1,0.05,0.01$ (from top left to bottom right).

$64, N_{\tau}=16, N_{p}=1638400$ and consider different values for $\varepsilon$. In Figure 11, the time history of $\xi$ defined by (3.3) is displayed for different values of $\varepsilon(\varepsilon=1,0.1,0.05,0.01)$. Different behaviors can be observed (see also [10]): when $\varepsilon$ is small, the Kelvin-Helmoltz instabilities occurs (up to $t \approx 20$ ) whereas when $\varepsilon$ is large, we do not get instability anymore. Note that all the numerical parameters are fixed and only the physical parameter $\varepsilon$ changes.

Furthermore, for large times it turns out that the two-scale $t$ and $\tau$ may no longer be separated variables and numerical instabilities may occur. These instabilities have been observed typically for $\varepsilon=0.1$ in our numerical tests. To avoid this, we propose to "re-initialize" the numerical solution every $t_{0}$ (where $t_{0}$ has to be chosen, typically $t_{0}=20$ for this test) as follows. At time $t=t_{0}$, we compute the original unknowns $x\left(t_{0}\right)$, $v\left(t_{0}\right)$ according to $(2.15)$ then we apply the general procedure where the initial time is now $t_{0}$ : two-scale formulation combined with the suitable preparation (2.26) of the initial data at $t=t_{0}$, in which $u_{ \pm}^{0}$ are now computed from $x\left(t_{0}\right), v\left(t_{0}\right)$ instead of $x(0), v(0)$. This refreshing process is repeated at all times $t=k t_{0}$, $k=1,2, \ldots$ To illustrate the influence of this strategy, we plot in Figure 12 the time history of $\xi$ defined by (3.3). The numerical parameters are the same as previously. Without the refreshing process, we observe that numerical instabilities are developed at $t \approx 80$ for the UA PIC approach. However, if we apply the refreshing process every $t_{0}=20$, the solution is smoothed and the numerical instabilities disappear. 

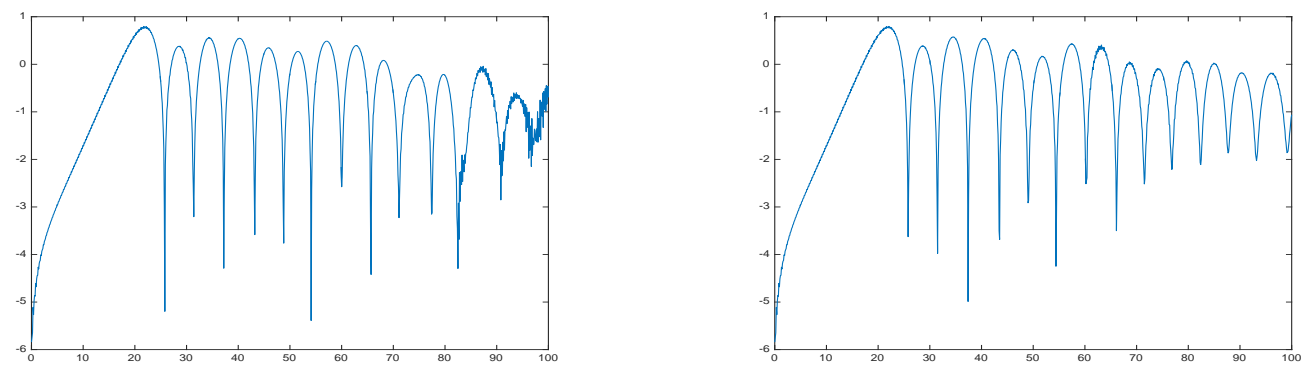

Figure 12: Time history the quantity $\xi(t)(3.3)$ for $\varepsilon=0.1$ without refreshing (left) and with refreshing every $t_{0}=20$ (right).

\section{Conclusion}

In this work, a two-scale Particle-In-Cell solver is introduced for solving the long time behavior of the four dimensional phase space Vlasov-Poisson system under the influence of a strong homogeneous magnetic field. In the asymptotic regime, the solution exhibits oscillations in time of period of order $\varepsilon^{2}$ where $1 / \varepsilon$ is related to the magnitude of the magnetic field.

This numerical scheme is based on a two-scale formulation in which the slow and fast time scale are separated, and on a suitable choice of the initial data for the two-scale system. Then, this solver is proved to be uniformly accurate of second order (in time) with respect to the parameter $\varepsilon \in(0,1]$. Numerical results confirm the good behavior of the scheme in different regimes and considering isotropic and non isotropic initial conditions.

The strategy is able to handle other scaling relevant for strongly magnetized plasmas such as the so-called finite Larmor radius regime. However the case of a non homogeneous magnetic field is still an issue, since oscillations in space and velocity have to be considered.

\section{Acknowledgements}

This work was supported by the French ANR project MOONRISE ANR-14-CE23-0007-01. N. Crouseilles and M. Lemou are supported by the Enabling Research EUROFusion project CfP-WP14-ER-01/IPP-03.

\section{References}

[1] C.K. Birdsall, A.B. Langdon, Plasma Physics via Computer Simulation, Adam Hilger, 1991.

[2] M. Bostan, The Vlasov-Maxwell system with strong initial magnetic field. Guiding-center approximation, SIAM J. Multiscale Model. Simul., Vol. 6, No. 3, pp.1026-1058 (2007)

[3] Ph. Chartier, N. Crouseilles, M. Lemou, F. Méhats, Uniformly accurate numerical schemes for highly oscillatory Klein-Gordon and nonlinear Schrödinger equations, Numer. Math. 129 (2015) pp. 211-250.

[4] N. Crouseilles, S. Jin, M. Lemou, Nonlinear Geometric Optics method based multi-scale numerical schemes for highlyoscillatory transport equations, hal.

[5] N. Crouseilles, M. Lemou, F. MÉhats, Asymptotic preserving schemes for highly oscillatory Vlasov-Poisson equations, J. Comput. Phys. 248 (2013) pp. 287-308.

[6] N. Crouseilles, M. Lemou, F. MÉhats, X. Zhao, Uniformly accurate forward semi-Lagrangian methods for highly oscillatory Vlasov-Poisson equations, preprint, hal-01286947 (2016). 
[7] N. Crouseilles, M. Mehrenberger, E. SonnendrüCker, Conservative semi-Lagrangian schemes for Vlasov equations, J. Comput. Phys. 229 (2010) pp. 1927-1953.

[8] P. Degond, P.A. Raviart, The paraxial approximation of the Vlasov-Maxwell equations, Math. Models Methods Appl. Sci. 3 (1993) pp. 513-562.

[9] D. H. E. Dubin, J. A. Krommes, C. Oberman, W. W. Lee, Nonlinear gyrokinetic equations, Phys. Fluids, 26 (1983).

[10] F. Filbet, M. Rodrigues, Asymptotically stable particle-in-cell methods for the Vlasov-Poisson system with a strong external magnetic field, SIAM J. Numer. Analysis, (2016).

[11] F. Filbet, E. SonnendrüCKer, Modeling and numerical simulation of space charge dominated beams in the paraxial approximation, Math. Models Methods Appl. Sci. 16 (2005) pp. 763-791.

[12] E. Frénod, S.A. Hirstoaga, M. Lutz, E. Sonnendrücker, Long time behavior of an exponential integrator for a Vlasov-Poisson system with strong magnetic field, Commun. in Comput. Phys. 18 (2015) pp. $263-296$.

[13] E. Frénod, F. Salvarani And E. Sonnendrücker, Long time simulation of a beam in a periodic focusing channel via a two-scale PIC-method, Math. Models Methods Appl. Sci. 19 (2009) pp. 175-197.

[14] E. FrÉnod, E. SonnendrüCKER, Long time behavior of the two-dimensional Vlasov equation with a strong external magnetic field, Math. Models Methods Appl. Sci. 10 (2000) pp. 539-553.

[15] G.B. Jacobs, J.S. Hesthaven, Implicit-Explicit time integration of a high-order particle-in-cell method with hyperbolic divergence cleaning, Comput. Phys. Comm. 180 (2009) pp. 1760-1767.

[16] S. JIN, Efficient asymptotic-preserving (AP) schemes for some multiscale kinetic equations, SIAM J. Sci. Comput. 21 (1999) pp. 441-454.

[17] M. Lemou, F. MÉhats, X. Zhao, Uniformly accurate numerical schemes for the nonlinear Dirac equation in the nonrelativistic limit regime, preprint, arXiv: 1605.02475[math.NA] (2016).

[18] W. W. LeE, Gyrokinetic approach in particle simulation, Phys. Fluids 26 (1983).

[19] A. Mouton, Two-scale semi-Lagrangian simulation of a charged particles beam in a periodic focusing channel, Kinet. Relat. Models 2 (2009) pp. 251-274.

[20] L. Saint-Raymond, The gyro-kinetic approximation for the Vlasov-Poisson system, Math. Models Methods Appl. Sci. 10, pp. $13051332(2000)$.

[21] M. Shoucri, A two-level implicit scheme for the numerical solution of the linearized vorticity equation, Internat. J. Numer. Methods Engrg. 17 (1981) pp. 1525-1538.

[22] E. SonnendrüCKer, Numerical Methods for Vlasov-Maxwell Equations, Springer, 2016. 